\title{
The Log Generalized Lindley-Weibull Distribution with Application
}

\author{
Broderick O. Oluyede ${ }^{1}$, Fedelis Mutiso ${ }^{2}$, Shujiao Huang ${ }^{3}$ \\ ${ }^{1}$ Department of Mathematical Sciences, Georgia Southern University \\ ${ }^{2}$ Department of Biostatistics, University of Washington \\ ${ }^{3}$ Department of Mathematics, University of Houston
}

\begin{abstract}
A new distribution called the log generalized Lindley-Weibull (LGLW) distribution for modeling lifetime data is proposed. This model further generalizes the Lindley distribution and allows for hazard rate functions that are monotonically decreasing, monotonically increasing and bathtub shaped. A comprehensive investigation and account of the mathematical and statistical properties including moments, moment generating function, simulation issues and entropy are presented. Estimates of model parameters via the method of maximum likelihood are given. Real data examples are presented to illustrate the usefulness and applicability of this new distribution.
\end{abstract}

Key words: Lindley distribution; Lindley-Weibull distribution; Maximum likelihood estimation

\section{Introduction}

The continuous one parameter Lindley distribution was introduced by Lindley (1958). Lindley used the distribution named after him to illustrate a difference between fiducial distribution and posterior distribution. Lindley distribution with the probability density function (pdf)

$$
\mathrm{f}(\mathrm{x} ; \theta)=\frac{\theta^{2}(1+x) \exp (-\theta x)}{1+\theta}, x>0, \theta>0,
$$

is a two-component mixture of an exponential distribution with scale parameter $\theta$ and gamma distribution with shape parameter 2 and scale parameter $\theta$. The mixing proportion is $\mathrm{p}=$ $\theta /(\theta+1)$. Sankaran $(1970)$ derived the Poisson-Lindley distribution. In this case, Lindley distribution was chosen as the mixing distribution when the parameter of the Poisson distribution is considered random. The resulting Poisson-Lindley distribution provided a better fit to the empirical set of data considered than the negative binomial and Hermite distributions. Recently, Ghitany et al. $(2008,2011)$ studied various properties of Lindley distribution and the twoparameter weighted Lindley distribution with applications to survival data. Bakouch et al. (2012) introduced an extension of the Lindley distribution that offers more flexibility in the modeling of lifetime data. Ghitany et al. (2013) presented results on the two-parameter generalization referred to as the power Lindley distribution. See Krishna and Kumar (2011) for additional results on reliability estimation of the Lindley distribution with progressive type II censored sample. 
Because of having only one parameter, the Lindley distribution does not provide enough flexibility for analyzing different types of lifetime data. To increase the flexibility for modeling purposes it will be useful to consider further generalizations of this distribution. This paper offers a five-parameter family of distributions which generalizes the Lindley distribution.

There are several ways of generalizing a continuous distribution $\mathrm{G}(\mathrm{x})$, and they include Kumaraswamy-G, beta-G, McDonald-G, and gamma-G to mention a few. Kumaraswamy (1980) distribution is given by

$$
\mathrm{G}_{\mathrm{k}}(\mathrm{x})=1-\left(1-\mathrm{x}^{\psi}\right)^{\phi}, 0 \leq x \leq 1,
$$

for $\psi>0$ and $\phi>0$. Replacing $x$ by $G(x)$ on the right hand side of the equation gives the Kumaraswamy-G family:

$$
\mathrm{G}_{\mathrm{KG}}(x)=1-\left(1-G^{\psi}(x)\right)^{\phi} .
$$

The beta-G family of distributions (Lee et al., 2007, Famoye et al., 2005) among others is given by

$$
\mathrm{G}_{\mathrm{BG}}(x)=\frac{1}{B(a, b)} \int_{0}^{G(x)} w^{a-1}(1-w)^{b-1} d w,
$$

for $\mathrm{a}>0$ and $\mathrm{b}>0$. The McDonald-G family of distributions (Cordeiro et al., 2012) is given by

$$
\mathrm{G}_{\mathrm{McG}}(x)=\frac{1}{B\left(a c^{-1}, b\right)} \int_{0}^{G^{c}(x)} w^{a c^{-1}-1}(1-w)^{b-1} d w,
$$

for $\mathrm{a}, \mathrm{b}$ and $\mathrm{c}>0$. The Gamma-G family of distributions (Zografos and Balakrishnan, 2009, Pinho et al., 2012) is

$$
\mathrm{G}_{\mathrm{GG}}(x)=\frac{\gamma\left(-\theta^{-1} \log (\bar{G}(x)), \alpha\right)}{\Gamma(\alpha)}
$$

for $\alpha, \theta>0$, where $\bar{G}(\mathrm{x})=1-\mathrm{G}(\mathrm{x})$.

We consider a further generalization of the generalized Lindley distribution via the T-X family of distributions proposed by Alzaatreh et al. (2013) to obtain the cumulative distribution function (cdf) of the log generalized Lindley-Weibull distribution. The generalization (Alzaatreh et al., 2013) is given by the following cdf:

$$
\mathrm{G}(\mathrm{x})=\int_{0}^{\mathrm{W}(\mathrm{F}(\mathrm{x}))} k(y) d y,
$$

where $0<\mathrm{W}(\mathrm{F}(\mathrm{x}))<\infty$, is a nondecreasing function of $\mathrm{x}, \mathrm{k}($.$) is taken to be the$ generalized Lindley distribution of Zakerzadeh and Dolati (2009) and F(x)is the Weibull cdf. The corresponding pdf $\mathrm{g}$, is given by

where $\mathrm{W}(\mathrm{F}(\mathrm{x}))=-\ln (1-\mathrm{F}(\mathrm{x}))$.

$$
\mathrm{g}(\mathrm{x})=\frac{f(x)}{\bar{F}(x)} k(W(F(x)))
$$


The main objective of this article is to construct and explore the properties of the fiveparameter log generalized Lindley-Weibull (LGLW) distribution. The beauty of this model is the fact that it not only generalizes the generalized Lindley distribution but also exhibits the desirable properties of increasing, decreasing, and bathtub shaped hazard function.

The model provides a better fit to data in the sense that it leads to more accurate results and prediction, which should facilitate better public policy in a wide range of areas including but not limited to medicine and environmental health, genetics, reliability, survival analysis and time-to event data analysis.

The outline of this paper is as follows: In section 2 some generalized Lindley distributions including the new LGLW distribution are introduced. This section also includes some properties such as the behavior of the hazard function, reverse hazard function and sub-models of the log generalized Lindley-Weibull distribution. Section 3 contains the moment generating function, moments, distribution of functions of log generalized Lindley-Weibull random variables and simulation. Measures of uncertainty are given in section 4. Section 5 contains the estimation of parameters via the maximum likelihood estimation technique. Fisher information and asymptotic confidence intervals are also presented in section 5. We end with applications in section 6 and concluding remarks in section 7 .

\section{Generalizations of the Lindley Distribution}

In this section, we present further generalizations of the Lindley distribution. First, we discuss some generalizations that are in the literature, or in preparation.

\subsection{Generalized Lindley Distribution}

Let $V_{1}$ and $V_{2}$ be two independent random variables distributed according to gamma $(\alpha, \theta)$ and gamma $(\alpha+1, \theta)$, respectively. That is, $\mathrm{V}_{1} \sim \operatorname{GAM}(\alpha, \theta)$ and $\mathrm{V}_{2} \sim \operatorname{GAM}(\alpha+1, \theta)$. For $\beta \geq 0$, consider the random variable $\mathrm{X}=\mathrm{V}_{1}$ with probability $\frac{\theta}{\theta+\beta}$, and $\mathrm{X}=\mathrm{V}_{2}$ with probability $\frac{\beta}{\theta+\beta}$. It is easy to verify that the density function of $\mathrm{X}$ is given by

$$
\mathrm{f}_{\mathrm{GL}}(x ; \alpha, \theta, \beta)=\frac{\theta}{\theta+\beta} f_{g_{\alpha}}(x ; \alpha, \theta)+\frac{\beta}{\theta+\beta} f_{g_{\alpha+1}}(x ; \alpha+1, \theta)
$$

which may be written as

$$
\mathrm{f}_{\mathrm{GL}}(x ; \alpha, \theta, \beta)=\frac{\theta^{2}(\theta x)^{\alpha-1}(\alpha+\beta x) e^{-\theta x}}{(\theta+\beta) \Gamma(\alpha+1)}, x>0, \beta, \alpha, \theta>0
$$

where $\mathrm{f}_{\mathrm{g}_{\alpha}}(x)$ is the gamma pdf with parameters $\alpha$ and $\theta$, that is,

$$
\mathrm{f}_{\mathrm{g}_{\alpha}}(x ; \alpha, \theta)=\frac{\theta^{\alpha} x^{\alpha-1} e^{-\theta x}}{\Gamma(\alpha)},
$$

for $\mathrm{x}>0, \alpha, \theta>0$. See Zakerzadeh and Dolati, (2009) for additional details. The distribution contains the Lindley distribution as particular case, where $\alpha=\beta=1$. When $\beta=0$, equation (3) reduces to the density function of the gamma distribution with the parameters $\alpha$ and $\theta$. The case 
$\alpha=1$ and $\beta=0$, reduces to ordinary exponential distribution. In general, if $\mathrm{V}_{\mathrm{i}} \sim G A M\left(\alpha_{i}, \theta_{i}\right), i=1,2, \ldots$, and $\mathrm{X}=\mathrm{v}_{\mathrm{i}}$ with probability $\mathrm{p}_{\mathrm{i}}$, and $\sum_{i} p_{i}=1$, then

$$
\mathrm{f}_{\mathrm{X}}(x)=\sum_{i} p_{i} \frac{\theta_{i}}{\Gamma\left(\alpha_{i}\right)} x^{\alpha_{i}-1} e^{-\theta_{i} x} I_{(0, \infty)}(x)
$$

Clearly, the generalized Lindley (GL) distribution is a special case of (5).

\subsection{Exponentiated Lindley Distribution}

A generalization of the Lindley distribution due to Nadarajah et al. (2011) is the two parameter Exponentiated Lindley distribution with cumulative distribution function (cdf) and probability density function (pdf) given by

and

$$
F_{G L}(x ; \theta, \alpha)=\left[1-\frac{1+\theta+\theta x}{1+\theta} e^{-\theta x}\right]^{\alpha},
$$

$$
\mathrm{f}_{\mathrm{GL}}(x ; \theta, \alpha)=\frac{\theta^{2} \alpha}{1+\theta}\left[1-\frac{1+\theta+\theta x}{1+\theta} e^{-\theta x}\right]^{\alpha-1}(1+x) e^{-\theta x},
$$

for $\mathrm{x}>0, \alpha>0$, and $\theta>0$, respectively.

\subsection{Beta-Generalized Lindley Distribution}

A further generalization of the Lindley distribution, although not studied in this paper is the beta-generalized Lindley (BGL) distribution, (Oluyede and Yang, 2014). The four parameter beta-generalized Lindley (BGL) cdf is given by

$$
\mathrm{F}_{\mathrm{BGL}}(x ; \alpha, \theta, a, b)=\frac{1}{B(a, b)} \int_{0}^{G(x ; \theta, \alpha)} t^{a-1}(1-t)^{b-1} d t,
$$

where $\mathrm{G}(\mathrm{x} ; \theta, \alpha)=\left\{1-\frac{1+\theta+\theta x}{1+\theta} e^{-\theta x}\right\}^{\alpha}$, for $\mathrm{x} \geq 0, \alpha>0, \theta>0, \mathrm{a}>0, \mathrm{~b}>0$. The corresponding pdf is given by

$$
\begin{aligned}
\mathrm{f}_{\mathrm{BGL}}(x ; \alpha, \theta, a, b)=\frac{\alpha \theta^{2}(1+x) e^{-\theta x}}{B(a, b)(1+\theta)}\left\{1-\frac{1+\theta+\theta x}{1+\theta} e^{-\theta x}\right\}^{a \alpha-1} \\
\times\left\{1-\left\{1-\frac{1+\theta+\theta x}{1+\theta} e^{-\theta x}\right\}^{\alpha}\right\}^{b-1}
\end{aligned}
$$

for $\mathrm{x} \geq 0, \alpha>0, \theta>0, \mathrm{a}>0, \mathrm{~b}>0$. If $\alpha=1$, we obtained the beta-Lindley (BL) distribution. If $\mathrm{a}=\mathrm{b}=\alpha=1$, we obtain the Lindley distribution. See Yang and Oluyede (2014) for additional details on the Exponentiated Kumaraswamy Lindley distribution.

\subsection{The Log Generalized Lindley-Weibull Distribution}


In this section, we introduce a new generalization of the Lindley distribution via the Weibull model and study its mathematical and statistical properties.

\subsubsection{Generalization-The Model}

Based on a continuous baseline cdf $\mathrm{F}(\mathrm{x})$ and survival function $\bar{F}(x)=1-F(x)$, with pdf $\mathrm{f}(\mathrm{x})$, Zografos and Balakrishnan (2009) defined the cdf

$$
G_{Z B}(x)=\frac{1}{\Gamma(\delta)} \int_{0}^{-\log (1-F(x))} t^{\delta-1} e^{-t} d t, \delta>0 \text { and } x \in \boldsymbol{R} .
$$

Along the same lines, Ristić and Balakrishnan (2011) proposed an alternative gammagenerator given by the cdf and pdf

and

$$
G_{R B}(x)=1-\frac{1}{\Gamma(\delta)} \int_{0}^{-\log (F(x))} t^{\delta-1} e^{-t} d t, \delta>0 \text { and } x \in \boldsymbol{R},
$$

$$
g_{R B}(x)=\frac{1}{\Gamma(\delta)}[-\log (F(x))]^{\delta-1} f(x), \delta>0 \text { and } x \in \boldsymbol{R} .
$$

Now, we consider a generalizations of the generalized Lindley distribution given by Zakerzadeh and Dolati (2009) via the Weibull distribution. The generalization is given by the following cdf (Alzaatreh et al., 2013):

$$
G_{L G L W}(x)=\int_{0}^{-\log \left(1-F_{W}(x)\right)} f_{G L}(y) d y,
$$

where $\mathrm{g}_{\mathrm{GL}}(x)$ is the generalized Lindley pdf and $\mathrm{F}_{\mathrm{W}}(x)$ is the Weibull cdf. The pdf of the $\log$ generalized Lindley-Weibull (LGLW) distribution is given by

$$
g_{L G L W}(x ; \alpha, \beta, \theta, \gamma, c)=\frac{f_{W}(x ; \gamma, c)}{\bar{F}_{W}(x ; \gamma, c)} f_{G L}\left(-\ln \left(1-F_{W}(x ; \gamma, c)\right) ; \alpha, \beta, \theta\right),(14
$$

where the survival function $\bar{F}_{W}(x ; \gamma, c)=1-F_{W}(x ; \gamma, c)=e^{-\left(\frac{x}{\gamma}\right)^{c}}$, for $\mathrm{x}>0, \gamma>0$, and $\mathrm{c}>0$. The well-known hazard function of the Weibull distribution is given by $\mathrm{h}_{\mathrm{W}}(x ; \gamma, c)=$ $\frac{f_{W}(x ; \gamma, c)}{F_{W}(x ; \gamma, c)}=\frac{c}{\gamma}\left(\frac{x}{\gamma}\right)^{c-1}$. It follows therefore that the five-parameter LGLW cdf is given by

$$
\begin{aligned}
G_{L G L W}(x)=\int_{0}^{\left(\frac{x}{\gamma}\right)^{c}} \frac{\theta^{2}(\theta y)^{\alpha-1}(\alpha+\beta y) e^{-\theta y}}{(\beta+\theta) \Gamma(\alpha+1)} d y \\
\quad=\frac{1}{(\beta+\theta) \Gamma(\alpha)}\left\{\theta[\Gamma(\alpha)-\Gamma(\alpha, u)]+\frac{\beta}{\alpha}[\Gamma(\alpha)-\Gamma(\alpha+1, u)]\right\},
\end{aligned}
$$

where $\Gamma(\mathrm{s}, \mathrm{x})=\int_{x}^{\infty} t^{s-1} e^{-t} d t$ is the upper incomplete gamma function and $\mathrm{u}=\theta\left(\frac{\mathrm{x}}{\gamma}\right)^{c}$. The corresponding pdf is given by

$$
g_{L G L W}(x ; \alpha, \theta, \beta, \gamma, c)=\frac{c \theta^{\alpha+1}}{\gamma(\beta+\theta) \Gamma(\alpha+1)}\left(\frac{x}{\gamma}\right)^{c \alpha-1}\left\{\alpha+\beta\left(\frac{x}{\gamma}\right)^{c}\right\} e^{-\theta\left(\frac{x}{\gamma}\right)^{c}},
$$

for $\mathrm{x}>0$, and $\theta, \alpha, c, \gamma, \beta>0$. The graphs of the LGLW pdf, $\mathrm{g}_{\mathrm{LGLW}}$ are given in Figure 1 for selected values of the parameters $\alpha, \theta, \beta, \gamma$, and c. Note that the parameters $\beta, \gamma$, and $\theta$ are scale parameters, and $\alpha, c$ are shape parameters. The graphs show that the pdf of the LGLW distribution can be right skewed or decreasing for the selected values of the model parameters. 


\subsubsection{Some LGLW Sub-models}

In this subsection, we present some sub-models of the LGLW distribution for selected values of the parameters $c, \alpha, \gamma, \beta$ and $\theta$.

If $\mathrm{c}=\gamma=1$, then $\operatorname{gLGLW}_{\mathrm{LG}}(x ; \alpha, \theta, \beta)=\frac{\theta^{\alpha+1} x^{\alpha-1}(\alpha+\beta x) e^{-\theta x}}{(\beta+\theta) \Gamma(\alpha+1)}$. This is the generalized

Lindley distribution, denoted by $\operatorname{GENLIN}(\alpha, \theta, \backslash$ beta $)$.

If $\mathrm{c}=\alpha=\gamma=\beta=1$, then $\operatorname{g}_{\mathrm{LGLW}}(x ; \theta)=\frac{\theta^{2}}{1+\theta}(1+x) e^{-\theta x}$, which is the Lindley distribution and is denoted by $\operatorname{LIN}(\theta)$ for $\mathrm{x}, \theta>0$.

If $\mathrm{c}=1$ and $\lambda=\frac{\theta}{\gamma}$, then $\mathrm{g}_{\mathrm{LGLW}}(x)=\frac{\gamma(\lambda x)^{\alpha}}{(\beta+\lambda \gamma) \Gamma(\alpha+1)}\left(\frac{\lambda}{x}\right)\left\{\alpha+\beta\left(\frac{x}{\gamma}\right)\right\} e^{-\lambda x}$.

If $\mathrm{c}=\alpha=\beta=1$, then $\mathrm{g}_{\mathrm{LGLW}}(\mathrm{x})=\frac{\theta^{2}}{\gamma(1+\theta)}\left(1+\frac{x}{\gamma}\right) e^{-\theta\left(\frac{x}{\gamma}\right)}$.

If $\alpha=\beta=1$, then $\operatorname{gLGLW}_{\mathrm{LLW}}(x)=\frac{c \theta^{2}}{\gamma(1+\theta)}\left(\frac{x}{\gamma}\right)^{c-1}\left\{1+\left(\frac{x}{\gamma}\right)^{c}\right\} e^{-\theta\left(\frac{x}{\gamma}\right)^{c}}$.

If $\gamma=\beta=1$, then $\operatorname{gLGLW}_{\mathrm{LLW}}(x)=\frac{c \theta^{\alpha+1}}{(1+\theta) \Gamma(\alpha+1)} x^{c \alpha-1}\left(\alpha+x^{c}\right) e^{-\theta x^{c}}$.

If $\mathrm{c}=1$, then $\mathrm{g}_{\mathrm{LGLW}}(x)=\frac{\theta^{\alpha+1}}{\gamma(\beta+\theta) \Gamma(\alpha+1)}\left(\frac{x}{\gamma}\right)^{\alpha-1}\left\{\alpha+\beta\left(\frac{x}{\gamma}\right)\right\} e^{-\theta\left(\frac{x}{\gamma}\right)}$.

If $\mathrm{c}=\alpha=1$, then $\operatorname{gLGLW}_{\mathrm{LL}}(x)=\frac{\theta^{2}}{\gamma(\beta+\theta)}\left\{1+\beta\left(\frac{x}{\gamma}\right)\right\} e^{-\theta\left(\frac{x}{\gamma}\right)}$.

If $\alpha=1$, then $\mathrm{g}_{\mathrm{LGLW}}(x)=\frac{c \theta^{2}}{\gamma(\beta+\theta)}\left(\frac{x}{\gamma}\right)^{c-1}\left\{1+\beta\left(\frac{x}{\gamma}\right)^{c}\right\} e^{-\theta\left(\frac{x}{\gamma}\right)^{c}}$.

\subsubsection{Shape}

For the LGLW pdf, the first derivative of $\log \left(\mathrm{g}_{\mathrm{LGLW}}(\mathrm{x})\right)$ is

$$
\frac{d}{d x} \ln \left(g_{L G L W}(x)\right)=-\frac{c \theta\left\{\left(\frac{x}{\gamma}\right)^{c}\right\}^{2} \beta+\{-c(\beta-\theta) \alpha-\beta(-1+c)\}\left(\frac{x}{\gamma}\right)^{c}-c \alpha^{2}+\alpha}{x\left\{\alpha+\beta\left(\frac{x}{\gamma}\right)^{c}\right\}}
$$

Therefore, $\mathrm{g}_{\mathrm{LGLW}}(\mathrm{x})$ has a unique model at $\mathrm{x}_{0}$, where $\mathrm{x}_{0}$ is the solution of the equation $\frac{d}{d x} \ln \left\{g_{L G L W}(x)\right\}=0$. That is,

which implies that

$$
-\frac{c \theta\left\{\left(\frac{x}{\gamma}\right)^{c}\right\}^{2} \beta+\{-c(\beta-\theta) \alpha-\beta(-1+c)\}\left(\frac{x}{\gamma}\right)^{c}-c \alpha^{2}+\alpha}{x\left\{\alpha+\beta\left(\frac{x}{\gamma}\right)^{c}\right\}}=0,
$$




$$
\mathrm{c} \theta\left\{\left(\frac{x}{\gamma}\right)^{c}\right\}^{2} \beta+\{-c(\beta-\theta) \alpha-\beta(-1+c)\}\left(\frac{x}{\gamma}\right)^{c}-c \alpha^{2}+\alpha=0 .
$$

Solving for $x$ in the above equation gives the mode. That is,

$$
x_{0}=\left[\frac{\{c(\beta-\theta) \alpha+\beta(-1+c)\}}{2 c \theta \beta}+\frac{\sqrt{\{-c(\beta-\theta) \alpha-\beta(-1+c)\}^{2}-4(\beta c \theta)\left(-c \alpha^{2}+\alpha\right)}}{2 c \theta \beta}\right]^{\frac{1}{c}} \gamma \text {. }
$$

Note that $\partial \ln \left(g_{L G L W}(x) / \partial x\right)<0 \Leftrightarrow x>x_{0}$ and $\partial \ln \left(\frac{g_{L G L W}(x)}{\partial x}\right)>0 \Leftrightarrow x<x_{0}$, where $x_{0}$ is given by equation (15) above. When $\alpha=\beta=\gamma=\mathrm{c}=1$,

$$
x_{0}=\frac{(1-\theta)+\sqrt{(\theta-1)^{2}}}{2 \theta}=\frac{(1-\theta)+\sqrt{(\theta+1)^{2}-4 \theta}}{2 \theta},
$$

which is the same result for the generalized Lindley distribution given by Zakerzadeh and Dolati (2009).

\subsubsection{Hazard and Reverse Hazard Functions}

In this section, we present the hazard and reverse hazard functions of the LGLW distribution. Graphs of the hazard function for selected values of the model parameters are given in Figure 2 and 3. The hazard and reverse hazard functions of the LGLW distribution are given by

$$
h(x)=\frac{g_{L G L W}(x)}{\bar{G}_{L G L W}(x)}=\frac{\frac{c \theta^{\alpha+1}}{\gamma(\beta+\theta) \Gamma(\alpha+1)}\left(\frac{x}{\gamma}\right)^{c \alpha-1}\left\{\alpha+\beta\left(\frac{x}{\gamma}\right)^{c}\right\} e^{-\theta\left(\frac{x}{\gamma}\right)^{c}}}{1-\frac{1}{(\beta+\theta) \Gamma(\alpha)}\left\{\theta[\Gamma(\alpha)-\Gamma(\alpha, u)]+\frac{\beta}{\alpha}[\Gamma(\alpha)-\Gamma(\alpha+1, u)]\right\}},
$$

and

$$
\tau_{\mathrm{LGLW}}(x)=\frac{g_{L G L W}(x)}{G_{L G L W}(x)}=\frac{c \theta^{\alpha+1}\left(\frac{x}{\gamma}\right)^{c \alpha-1}\left\{\alpha+\beta\left(\frac{x}{\gamma}\right)^{c}\right\} e^{-\theta\left(\frac{x}{\gamma}\right)^{c}}}{\gamma \alpha\left\{\theta[\Gamma(\alpha)-\Gamma(\alpha, u)]+\frac{\beta}{\alpha}[\Gamma(\alpha)-\Gamma(\alpha+1, u)]\right\}}
$$

for $\theta, \alpha, c, \gamma, \beta>0$, and $\mathrm{u}=\theta\left(\frac{x}{\gamma}\right)^{c}$, respectively. We obtain $\eta_{\mathrm{LGLW}}(x)=\frac{-g_{L G L W}^{\prime}(x)}{g_{L G L W}(x)}$ and $\eta_{L G L W}^{\prime}(x)$ and apply Glaser's Lemma (1980) to the LGLW pdf:

$\mathrm{g}_{\mathrm{LGLW}}(x)=\frac{c \theta^{\alpha+1}}{\gamma(\beta+\theta) \Gamma(\alpha+1)}\left(\frac{x}{\gamma}\right)^{c \alpha-1}\left\{\alpha+\beta\left(\frac{x}{\gamma}\right)^{c}\right\} \mathrm{e}^{-\theta\left(\frac{x}{\gamma}\right)^{c}}$,

for $\mathrm{x}>0, \mathrm{c}, \theta, \alpha, \gamma, \beta>0$. Note that

$$
\begin{aligned}
& \mathrm{g}_{L G L W}^{\prime}(x)= \frac{c \theta^{\alpha+1}\left(\frac{x}{\gamma}\right)^{c \alpha-1}(c \alpha-1)\left\{\alpha+\beta\left(\frac{x}{\gamma}\right)^{c}\right\} e^{-\theta\left(\frac{x}{\gamma}\right)^{c}}}{\gamma(\beta+\theta) \Gamma(\alpha+1) x} \\
&+\frac{c^{2} \theta^{\alpha+1}\left(\frac{x}{\gamma}\right)^{c \alpha-1} \beta\left(\frac{x}{\gamma}\right)^{c} e^{-\theta\left(\frac{x}{\gamma}\right)^{c}}}{\gamma(\beta+\theta) \Gamma(\alpha+1) x}
\end{aligned}
$$




$$
-\frac{c^{2} \theta^{\alpha+1}\left(\frac{x}{\gamma}\right)^{c \alpha-1}(c \alpha-1)\left\{\alpha+\beta\left(\frac{x}{\gamma}\right)^{c}\right\} \theta\left(\frac{x}{\gamma}\right)^{c} e^{-\theta\left(\frac{x}{\gamma}\right)^{c}}}{\gamma(\beta+\theta) \Gamma(\alpha+1) x}
$$

and

$$
\eta_{\mathrm{LGLW}}(x)=\frac{c \theta\left\{\left(\frac{x}{\gamma}\right)^{c}\right\}^{2} \beta+\{-c(\beta-\theta) \alpha-\beta(-1+c)\}\left(\frac{x}{\gamma}\right)^{c}-c \alpha^{2}+\alpha}{x\left\{\alpha+\beta\left(\frac{x}{\gamma}\right)^{c}\right\}} .
$$

When $\mathrm{c}=\gamma=1$, we have $\eta_{\mathrm{LGLW}}(x)=\frac{\alpha-\alpha^{2}-\alpha \beta x+\theta x(\alpha+\beta x)}{x(\alpha+\beta x)}$, which is the same result given by Zakerzadeh and Dolati (2009). Now,

$$
\begin{gathered}
\eta_{L G L W}^{\prime}(x)=\frac{2 c^{2} \theta\left\{\left(\frac{x}{\gamma}\right)^{c}\right\}^{2} \beta}{x^{2}\left\{\alpha+\beta\left(\frac{x}{\gamma}\right)^{c}\right\}}+\frac{\{-c(\beta-\theta) \alpha-\beta(-1+c)\}\left(\frac{x}{\gamma}\right)^{c} c}{x^{2}\left\{\alpha+\beta\left(\frac{x}{\gamma}\right)^{c}\right\}} \\
-\frac{c \theta\left\{\left(\frac{x}{\gamma}\right)^{c}\right\}^{2} \beta+\{-c(\beta-\theta) \alpha-\beta(-1+c)\}\left(\frac{x}{\gamma}\right)^{c}-c \alpha^{2}+\alpha}{x^{2}\left\{\alpha+\beta\left(\frac{x}{\gamma}\right)^{c}\right\}} \\
-\frac{\left\{c \theta\left\{\left(\frac{x}{\gamma}\right)^{c}\right\}^{2} \beta+\{-c(\beta-\theta) \alpha-\beta(-1+c)\}\left(\frac{x}{\gamma}\right)^{c}-c \alpha^{2}+\alpha\right\} \beta\left(\frac{x}{\gamma}\right)^{c} c}{x^{2}\left\{\alpha+\beta\left(\frac{x}{\gamma}\right)^{c}\right\}} .
\end{gathered}
$$

If $\mathrm{c}=\gamma=1$, then $\eta_{L G L W}^{\prime}(x)=\frac{\alpha^{3}+2 \alpha^{2} \beta x+\alpha \beta^{2} x^{2}-\alpha^{2}-2 \alpha \beta x}{x^{2}(\alpha+\beta x)^{2}}$, which is the same result obtained by Zakerzadeh and Dolati (2009). The graphs of the hazard and reverse hazard functions are given below for different values of parameters $\alpha, \theta, \beta, \gamma$, and c. For the selected values of the parameters $\alpha, \theta, \beta, \gamma$, and $c$, the graphs of the hazard function are decreasing, increasing and bathtub shaped.

\section{Moments and Distribution of Functions of Random Variables}

This section deals with the moment generating function, moments and related functions of LGLW distribution. The mean, standard deviation, coefficients of variation, skewness and kurtosis can be readily computed. Distributions of functions of the LGLW random variables are also presented.

\subsection{Moments}

In this section, we obtain the moments of the LGLW distribution and its sub-models. The $k^{t h}$ non-central moment for the LGLW distribution is 


$$
\mathrm{E}\left(\mathrm{x}^{\mathrm{k}}\right)=\int_{0}^{\infty} \frac{\gamma^{k} c \theta^{\alpha+1}}{\gamma(\beta+\theta) \Gamma(\alpha+1)}\left(\frac{x}{\gamma}\right)^{c \alpha+k-1}\left\{\alpha+\beta\left(\frac{x}{\gamma}\right)^{c}\right\} e^{-\theta\left(\frac{x}{\gamma}\right)^{c}} d x .
$$

Let $y=\left(\frac{x}{\gamma}\right)^{c}$, then $x=\gamma y^{\frac{1}{c}}$, and $\frac{\mathrm{dx}}{\mathrm{dy}}=\frac{1}{c} \gamma y^{\frac{1}{c}-1}$. Now,

$$
\mathrm{E}\left(\mathrm{X}^{\mathrm{k}}\right)=\frac{\gamma^{k} c \theta^{\alpha+1}}{(\beta+\theta) \Gamma(\alpha+1)}\left\{\alpha \int_{0}^{\infty} y^{\alpha+\frac{k}{c}-1} e^{-\theta y} d y+\beta \int_{0}^{\infty} y^{\alpha+\frac{k}{c}+1-1} e^{-\theta y} d y\right\} .
$$

Let $u=\theta y$, then $\frac{d u}{d y}=\theta$ and $d y=\frac{d u}{\theta}$, so that

$$
\begin{gathered}
\mathrm{E}\left(\mathrm{X}^{\mathrm{k}}\right)=\frac{\gamma^{k} \theta^{\alpha+1}}{(\beta+\theta) \Gamma(\alpha+1)} \\
\times\left\{\alpha \int_{0}^{\infty}\left(\frac{u}{\theta}\right)^{\alpha+\frac{k}{c}-1} e^{-u} \frac{d u}{\theta}+\beta \int_{0}^{\infty}\left(\frac{u}{\theta}\right)^{\alpha+\frac{k}{c}+1-1} \frac{d u}{\theta}\right\} \\
=\frac{\gamma^{k} \theta^{1-\frac{k}{c} \Gamma\left(\alpha+\frac{k}{c}\right)}}{(\beta+\theta) \Gamma(\alpha+1)}\left[\alpha+\beta \theta^{-1}\left(\alpha+\frac{k}{c}\right)\right] .
\end{gathered}
$$

The mean of LGLW distribution is

$$
\begin{aligned}
\mathrm{E}(\mathrm{X}) & =\frac{\gamma \theta^{1-\frac{1}{c}}}{(\beta+\theta) \Gamma(\alpha+1)} \Gamma\left(\alpha+\frac{1}{c}\right)\left\{\alpha+\beta \theta^{-1}\left(\alpha+\frac{1}{c}\right)\right\} . \\
\text { If } \mathrm{c}=1 \text {, then } \mathrm{E}(\mathrm{X}) & =\frac{\gamma}{\beta+\theta}\left\{\alpha+\beta \theta^{-1}(\alpha+1)\right\} .
\end{aligned}
$$

\subsection{Moment Generating Function}

Let $\mathrm{X}$ denote a random variable with $\operatorname{pdf} \operatorname{gLGLW}_{\mathrm{LG}}(x)$. The moment generating function (MGF) of $X, M(t)=E(\exp (t X))$, is given by

$$
\mathrm{M}_{\mathrm{X}}(\mathrm{t})=\sum_{j=0}^{\infty} \frac{t^{j} \theta^{1-\frac{j}{c}} \gamma^{j}}{j !(\beta+\theta) \Gamma(\alpha+1)}\left\{\alpha \Gamma\left(\alpha+\frac{j}{c}\right)+\beta \theta^{-1} \Gamma\left(\alpha+\frac{j}{c}+1\right)\right\} .
$$

Note that $\mathrm{M}_{\mathrm{X}}(t)=\sum_{j=0}^{\infty} \frac{t^{j}}{j !} E\left(X^{j}\right)$, where $\mathrm{E}\left(\mathrm{X}^{\mathrm{j}}\right)$ is given by equation (18).

\subsection{Distribution of Functions of Random Variables}

In this section, distributions of functions of random variables are presented. Recall the LGLW pdf is

$$
\mathrm{g}_{\mathrm{LGLW}}(x)=\frac{c \theta^{\alpha+1}}{\gamma(\beta+\theta) \Gamma(\alpha+1)}\left(\frac{x}{\gamma}\right)^{c \alpha-1}\left\{\alpha+\beta\left(\frac{x}{\gamma}\right)^{c}\right\} e^{-\theta\left(\frac{x}{\gamma}\right)^{c}}
$$

for $\mathrm{x}>0, \alpha, \beta, \theta, \gamma, \mathrm{c}>0$.

Pdf of $\mathrm{Y}=\left(\frac{X}{\gamma}\right)^{\mathrm{c}}$ : Let $\mathrm{y}=\left(\frac{\mathrm{x}}{\gamma}\right)^{c}$, then $\mathrm{x}=\gamma \mathrm{y}^{\frac{1}{c}}$ and $\frac{\mathrm{dx}}{\mathrm{dy}}=\frac{1}{c} \gamma y^{\frac{1}{c}-1}$. The pdf of $\mathrm{Y}=\left(\frac{\mathrm{X}}{\gamma}\right)^{\mathrm{c}}$ is given by 


$$
\mathrm{f}_{\mathrm{Y}}(y)=\frac{\theta^{\alpha+1}}{(\beta+\theta) \Gamma(\alpha+1)} y^{\alpha-1}(\alpha+\beta y) e^{-\theta y},
$$

for $y>0, \alpha, \beta, \theta>0$, which is the $\operatorname{GENLIN}(\alpha, \theta, \beta)$.

Pdf of $\mathrm{W}=\mathrm{X}^{\mathrm{c}}$ : Let $\mathrm{W}=\mathrm{x}^{\mathrm{c}}$, then $\mathrm{x}=\mathrm{w}^{\frac{1}{c}}$ and $\frac{\mathrm{dx}}{\mathrm{dw}}=\frac{1}{c} w^{\frac{1}{c}-1}$. The pdf of $\mathrm{W}=\mathrm{X}^{\mathrm{c}}$ is given by

$$
\mathrm{f}_{\mathrm{W}}(w)=\frac{\theta^{\alpha+1}}{\gamma(\beta+\theta) \Gamma(\alpha+1)} \frac{w^{\alpha-1}}{\gamma^{c \alpha-1}}\left\{\alpha+\beta\left(\frac{w}{\gamma^{c}}\right)\right\} e^{-\theta\left(\frac{w}{\gamma^{c}}\right)},
$$

for $\mathrm{w}>0, \alpha, \theta, \beta, \gamma, \mathrm{c}>0$. If $\gamma=1$, the two pdf's above are the same.

Pdf of $\mathrm{V}=\theta\left(\frac{\mathrm{X}}{\gamma}\right)^{\mathrm{c}}$ : Let $\mathrm{v}=\theta\left(\frac{\mathrm{x}}{\gamma}\right)^{\mathrm{c}}$, then $\mathrm{x}=\gamma\left(\frac{\mathrm{v}}{\theta}\right)^{\frac{1}{c}}$, and $\frac{\mathrm{dx}}{\mathrm{dv}}=\frac{\gamma}{c}\left(\frac{v}{\theta}\right)^{\frac{1}{c}-1} \frac{1}{\theta}$. The pdf of $\mathrm{V}=$ $\theta\left(\frac{X}{\gamma}\right)^{c}$ is given by

$$
\mathrm{f}_{\mathrm{V}}(v)=\frac{\theta^{\alpha}}{(\beta+\theta) \Gamma(\alpha+1)}\left(\frac{v}{\theta}\right)^{\alpha-1}\left\{\alpha+\beta\left(\frac{v}{\theta}\right)\right\} e^{-v},
$$

for $\mathrm{v}>0, \alpha, \theta, \gamma, \beta>0$.

\subsection{Simulation}

The density of generalized Lindley (GL) distribution can be written in terms of the gamma density function as

$$
\mathrm{f}(\mathrm{x} ; \alpha, \theta, \beta)=\frac{\theta}{\beta+\theta} f_{g}(x ; \alpha, \theta)+\frac{\beta}{\beta+\theta} f_{g}(x ; \alpha+1, \theta)
$$

To generate a random data $X_{i}, i=1, \ldots, n$, from $\operatorname{GL}(\alpha, \theta, \beta)$, Zakerzadeh and Dolati (2009), provided the following algorithm;

1. Generate $\mathrm{U}_{\mathrm{i}}, i=1, \ldots, n$, from $\mathrm{U}(0,1)$ distribution.

2. Generate $\mathrm{V}_{1 i}, i=1, \ldots, n$, from the $\operatorname{gamma}(\alpha, \theta)$.

3. Generate $\mathrm{V}_{2 i}, i=1, \ldots, n$, from the gamma $(\alpha+1, \theta)$.

4. If $\mathrm{U}_{\mathrm{i}} \leq \frac{\theta}{\beta+\theta}$, then set $\mathrm{X}_{\mathrm{i}}=V_{1 i}$; otherwise set $\mathrm{X}_{\mathrm{i}}=V_{2 i}, i=1, \ldots, n$.

Now given $\gamma$ and $\mathrm{c}$, we can generate random data $\mathrm{Y}_{\mathrm{i}}, i=1, \ldots, n$ where $\mathrm{Y}_{\mathrm{i}}=$ $\gamma X_{i}^{\frac{1}{c}} \sim \operatorname{LGLW}(\alpha, \beta, \theta, \gamma, c)$.

\section{Uncertainty Measures}

The concept of entropy plays a vital role in information theory. The entropy of a random variable is defined in terms of its probability distribution and can be shown to be a good measure of randomness or uncertainty. In this section, we present Renyi entropy, generalized entropy and s-entropy for the LGLW distribution. 


\subsection{Generalized Entropy}

Generalized entropy (GE) is widely used to measure inequality trends and differences. It is primarily used in income distributions. Kleiber and Kotz (2003) derived Theil index for GB2 distribution and Singh-Maddala model. The generalized entropy (GE) I $\left(\alpha^{*}\right)$ is defined as:

where

$$
\mathrm{I}\left(\alpha^{*}\right)=\frac{v_{\alpha^{*}} \mu^{-\alpha^{*}}-1}{\alpha^{*}\left(\alpha^{*}-1\right)}, \alpha^{*} \neq 0,1,
$$

$v_{\alpha^{*}}=E\left(X^{\alpha^{*}}\right)=\int_{0}^{\infty} x^{\alpha^{*}} g_{L G L W}(x) d x$

$=\frac{\gamma^{\alpha^{*}} \theta^{1-\frac{\alpha^{*}}{c}}}{(\beta+\theta) \Gamma(\alpha+1)} \Gamma\left(\alpha+\frac{\alpha^{*}}{c}\right)\left\{\alpha+\beta \theta^{-1}\left(\alpha+\frac{\alpha^{*}}{c}\right)\right\}$,

and

$$
\mu=\frac{\gamma \theta^{1-\frac{1}{c}}}{(\beta+\theta) \Gamma(\alpha+1)} \Gamma\left(\alpha+\frac{1}{c}\right)\left\{\alpha+\beta \theta^{-1}\left(\alpha+\frac{1}{c}\right)\right\} .
$$

The bottom-sensitive index is $\mathrm{I}(-1)$, and the top-sensitive index is $\mathrm{I}(2)$. The mean logarithmic deviation (MLD) index is given by:

where

$$
\mathrm{I}(0)=\lim _{\alpha^{*} \rightarrow 0} I\left(\alpha^{*}\right)=\log (\mu)-v_{0}
$$

$$
\begin{gathered}
v_{0}=\int_{0}^{\infty} \log (x) d G_{L G L W}(x)=\int_{0}^{\infty}(\log (x)) g_{L G L W}(x) d x \\
=\int_{0}^{\infty} \frac{c \theta^{\alpha+1}}{\gamma(\beta+\theta) \Gamma(\alpha+1)}(\log (x))\left(\frac{x}{\gamma}\right)^{c \alpha-1}\left\{\alpha+\beta\left(\frac{x}{\gamma}\right)^{c}\right\} e^{-\theta\left(\frac{x}{\gamma}\right)^{c}} d x .
\end{gathered}
$$

Let $\mathrm{y}=\theta\left(\frac{x}{\gamma}\right)^{c}$, then $\log (x)=\log (\gamma)-\frac{1}{c} \log (\theta)+\frac{1}{c} \log (y)$. Now, we have

$$
\begin{aligned}
v_{0}= & \frac{\theta}{(\beta+\theta) \Gamma(\alpha+1)} \int_{0}^{\infty}\left\{\log (\gamma)+\frac{1}{c} \log (y)-\frac{1}{c} \log (\theta)\right\} y^{\alpha-\frac{1}{c}}\left\{\alpha+\beta \theta^{-1} y\right\} e^{-y} d y \\
= & \frac{\theta}{(\beta+\theta) \Gamma(\alpha+1)}\left[\left\{\log \left(\frac{\gamma}{\theta^{\frac{1}{c}}}\right) \alpha \Gamma\left(\alpha-\frac{1}{c}+1\right)+\frac{\alpha}{c} \Gamma^{\prime}\left(\alpha-\frac{1}{c}+1\right)\right\}\right. \\
& \left.+\left\{\log \left(\frac{\gamma}{\theta^{\frac{1}{c}}}\right) \beta \theta^{-1} \Gamma\left(\alpha-\frac{1}{c}+2\right)+\frac{\beta \theta^{-1}}{c} \Gamma^{\prime}\left(\alpha-\frac{1}{c}+2\right)\right\}\right] .
\end{aligned}
$$

Therefore, the MLD index reduces to

$$
\begin{aligned}
& \mathrm{I}(0)=\log (\gamma)+\left(1-\frac{1}{c}\right) \log (\theta)-\log (\beta+\theta)-\log (\Gamma(\alpha+1)) \\
& +\log \left(\Gamma\left(\alpha+\frac{1}{c}\right)\right)+\log \left\{\alpha+\beta \theta^{-1}\left(\alpha+\frac{1}{c}\right)\right\}
\end{aligned}
$$




$$
\begin{gathered}
-\frac{\theta}{(\beta+\theta) \Gamma(\alpha+1)}\left[\left\{\log \left(\frac{\gamma}{\theta^{\frac{1}{c}}}\right) \alpha \Gamma\left(\alpha-\frac{1}{c}+1\right)+\frac{\alpha}{c} \Gamma^{\prime}\left(\alpha-\frac{1}{c}+1\right)\right\}-\left\{\log \left(\frac{\gamma}{\theta^{\frac{1}{c}}}\right) \beta \theta^{-1} \Gamma\left(\alpha-\frac{1}{c}+2\right)+\right.\right. \\
\left.\left.\frac{\beta \theta^{-1}}{c} \Gamma^{\prime\left(\alpha-\frac{1}{c}+2\right)}\right\}\right],
\end{gathered}
$$

and Theil index is:

$$
\begin{aligned}
& \mathrm{I}(1)=\lim _{\alpha^{*} \rightarrow 1} I\left(\alpha^{*}\right)=\frac{\mu}{v_{1}}-\log (\mu) \\
& =\frac{\theta \frac{\alpha^{*}-1}{c} \Gamma\left(\alpha+\frac{1}{c}\right)\left\{\alpha+\beta \theta^{-1}\left(\alpha+\frac{1}{c}\right)\right\}}{\Gamma\left(\alpha+\frac{\alpha^{*}}{c}\right)\left\{\alpha+\beta \theta^{-1}\left(\alpha+\frac{\alpha^{*}}{c}\right)\right\}}-\log (\gamma) \\
& +\left(1-\frac{1}{c}\right) \log (\theta)-\log (\beta+\theta)-\log (\Gamma(\alpha+1)) \\
& +\log \left(\Gamma\left(\alpha+\frac{1}{c}\right)\right)+\log \left\{\alpha+\beta \theta^{-1}\left(\alpha+\frac{1}{c}\right)\right\} .
\end{aligned}
$$

The generalized entropy for the sub-models can be readily obtained as well.

\subsection{Renyi Entropy}

An entropy of a random variable $X$ is a measure of variation of the uncertainty. A popular entropy measure is Rényi entropy (1961). If X has the pdf $\mathrm{f}($.$) , then Rényi entropy is defined by$ $\mathrm{I}_{\mathrm{R}}(b)=\frac{1}{1-b} \log \left(\int_{0}^{\infty} g^{b}(x) d x\right)$, where $\mathrm{b}>0$ and $\mathrm{b} \neq 1$. Suppose $\mathrm{X}$ has the LGLW pdf, then for any real number $\mathrm{b}>0$, and $\mathrm{b} \neq 1$,

$$
\begin{aligned}
& \int_{0}^{\infty} g_{L G L W}^{b}(x) d x=\int_{0}^{\infty}\left[\left\{\frac{c \theta^{\alpha+1}\left\{\alpha+\beta\left(\frac{x}{\gamma}\right)^{c}\right\}}{\gamma(\beta+\theta) \Gamma(\alpha+1)}\right\}^{b}\left(\frac{x}{\gamma}\right)^{b(c \alpha-1)} e^{\left.-\theta b\left(\frac{x}{\gamma}\right)^{c}\right]} d x\right. \\
= & \sum_{j=0}^{\infty}\left\{\frac{c \theta^{\alpha+1}}{\gamma(\beta+\theta) \Gamma(\alpha+1)}\right\}^{b} \frac{b !}{j !(b-j) !} \beta^{j} \alpha^{b-j} \int_{0}^{\infty}\left(\frac{x}{\gamma}\right)^{b(c \alpha-1)+c j} e^{-\theta b\left(\frac{x}{\gamma}\right)^{c}} d x .
\end{aligned}
$$

Let $v=\theta b\left(\frac{x}{\gamma}\right)^{c}$, and $\frac{\mathrm{dx}}{\mathrm{d} v}=\frac{1}{c}\left(\frac{v}{\theta b}\right)^{\frac{1}{c}-1} \frac{\gamma}{\theta b}$. Now, making the substitution, we have

$$
\begin{aligned}
& \int_{0}^{\infty} g_{L G L W}^{b}(x) d x=\left\{\frac{c \theta^{\alpha+1}}{\gamma(\beta+\theta) \Gamma(\alpha+1)}\right\}^{b} \sum_{j=0}^{\infty} \frac{b !}{j !(b-j) !} \beta^{j} \alpha^{b-j} \frac{\gamma}{c} \\
& \times(\theta b)^{-b \alpha+\frac{b}{c}-j-\frac{1}{c} \Gamma\left(b \alpha-\frac{b}{c}+j+\frac{1}{c}\right) .}
\end{aligned}
$$

Now, for any real number $b>0$, and $b \neq 1$, Rényi entropy is given by

$$
\mathrm{I}_{\mathrm{R}}(b)=\frac{b}{1-b} \log \left(\frac{c \theta^{\alpha+1}}{\gamma(\beta+\theta) \Gamma(\alpha+1)}\right)
$$




$$
+\frac{1}{1-b} \log \left\{\sum_{j=0}^{\infty} \frac{b !}{j !(b-j) !}\left(\frac{\beta}{\alpha}\right)^{j} \alpha^{b} \frac{\gamma}{c}(\theta b)^{-b \alpha+\frac{b}{c}-j-\frac{1}{c}} \Gamma\left(b \alpha-\frac{b}{c}+j+\frac{1}{c}\right)\right\},
$$

for $\alpha, \beta, \gamma, c>0$. By taking the limit as b $\uparrow 1$ and using L'Hospital's rule, we obtain Shannon entropy (1948). Rényi entropy for the sub-models can be readily obtained.

\section{3 s-Entropy}

The s-entropy is a one parameter generalization of the Shannon entropy and is defined by

$$
\mathrm{H}_{\mathrm{s}}\left(g_{L G L W}\right)=\frac{1}{s-1}\left[1-\int_{0}^{\infty} g_{L G L W}^{s}(x) d x\right], s>0, \text { and } s \neq 1 \text {. }
$$

Now, if $s \in R_{+}$and $s \neq 1$,

$$
\mathrm{H}_{\mathrm{s}}\left(g_{L G L W}\right)=\frac{1}{s-1}\left[\begin{array}{c}
1-\sum_{j=0}^{\infty}\left\{\frac{c \theta^{\alpha+1}}{\gamma(\beta+\theta) \Gamma(\alpha+1)}\right\}^{s}\left(\begin{array}{c}
s \\
j
\end{array}\right) \beta^{j} \alpha^{s-j} \\
\times \int_{0}^{\infty}\left(\frac{x}{\gamma}\right)^{s(c \alpha-1)+c j} e^{-\theta s\left(\frac{x}{\gamma}\right)^{c}} d x
\end{array}\right] .
$$

The integral in equation (26) follow directly from the result of the integral in Rényi entropy with $\mathrm{s}$ in place of $\mathrm{b}$. For $\mathrm{s}=1, \mathrm{H}_{\mathrm{s}}\left(g_{L G L W}\right)=-E\left[\log \left(g_{L G L W}(X)\right)\right]$, which is Shannon entropy (1948).

\section{Maximum Likelihood Estimation in the LGLW Distribution}

In this section, we obtain estimates of the parameters of the LGLW distribution. Methods of maximum likelihood (ML) estimation and asymptotic confidence intervals for the model parameters are presented.

\subsection{Maximum Likelihood Estimators}

Suppose $\mathrm{x}=\left(\mathrm{x}_{1}, \mathrm{x}_{2}, \ldots, \mathrm{x}_{\mathrm{n}}\right)$ is a random sample of size $\mathrm{n}$ from the LGLW distribution. The $\log$-likelihood function is given by:

$$
\begin{gathered}
l(\alpha, \beta, \gamma, \mathrm{c}, \theta)=\mathrm{n} \ln c+n(\alpha+1) \ln \theta-n \ln \gamma-n \ln (\beta+\theta) \\
-\mathrm{n} \ln \Gamma(\alpha+1)+n(-c \alpha+1) \ln \gamma+(c \alpha-1) \sum_{i=1}^{n} \ln x_{i} \\
+\sum_{i=1}^{n} \ln \left\{\alpha+\beta\left(\frac{x_{i}}{\gamma}\right)^{c}\right\}-\sum_{i=1}^{n} \theta\left(\frac{x_{i}}{\gamma}\right)^{c} .
\end{gathered}
$$

The partial derivatives of $l$ with respect to the parameters are:

$$
\frac{\partial l}{\partial \alpha}=n \ln \theta-\frac{n \Gamma^{\prime(\alpha+1)}}{\Gamma(\alpha+1)}-n c \ln \gamma+c \sum_{i=1}^{n} \ln x_{i}+\sum_{i=1}^{n} \frac{1}{\alpha+\beta\left(\frac{x_{i}}{\gamma}\right)^{c}}
$$




$$
\begin{aligned}
& \frac{\partial l}{\partial \beta}=-\frac{n}{\beta+\theta}+\sum_{i=1}^{n} \frac{\left(\frac{x_{i}}{\gamma}\right)^{c}}{\alpha+\beta\left(\frac{x_{i}}{\gamma}\right)^{c}} \\
& \frac{\partial l}{\partial \gamma}=-\frac{n}{\gamma}+\frac{n(-c \alpha+1)}{\gamma}-\sum_{i=1}^{n} \frac{\beta\left(\frac{x_{i}}{\gamma}\right)^{c} c}{\gamma\left\{\alpha+\beta\left(\frac{x_{i}}{\gamma}\right)^{c}\right\}}+\frac{1}{\gamma} \sum_{i=1}^{n} \theta\left(\frac{x_{i}}{\gamma}\right)^{c}, \\
& \frac{\partial l}{\partial \theta}=\frac{n(\alpha+1)}{\theta}-\frac{n}{\beta+\theta}-\sum_{i=1}^{n}\left(\frac{x_{i}}{\gamma}\right)^{c},
\end{aligned}
$$

and

$$
\frac{\partial l}{\partial c}=\frac{n}{c}-\operatorname{n} \alpha \ln \gamma+\sum_{\mathrm{i}=1}^{\mathrm{n}} \alpha \ln x_{i}+\sum_{i=1}^{n} \frac{\beta\left(\frac{x_{i}}{\gamma}\right)^{c} \ln \left\{\beta\left(\frac{x_{i}}{\gamma}\right)\right\}}{\left\{\alpha+\beta\left(\frac{x_{i}}{\gamma}\right)^{c}\right\}}-\sum_{i=1}^{n} \theta\left(\frac{x_{i}}{\gamma}\right)^{c} \ln \left\{\theta\left(\frac{x_{i}}{\gamma}\right)\right\} .
$$

The MLE of the parameters $\alpha, \beta, \gamma, \theta$ and $\mathrm{c}$, say $\hat{\alpha}, \hat{\beta}, \hat{\gamma}, \hat{\theta}$, and $\hat{c}$ are obtained by solving the equations $\frac{\partial l}{\partial \alpha}=0, \frac{\partial l}{\partial \beta}=0, \frac{\partial l}{\partial \gamma}=0, \frac{\partial l}{\partial \theta}=0$, and $\frac{\partial l}{\partial \mathrm{c}}=0$. There is no closed form solution, so these equations must be solved numerically to obtain the MLE of the parameters $\alpha, \theta, \beta, \gamma$ and c, Note that, if $\alpha, \beta, \gamma$ and $c$ are known, it follows from equation (29) that

$$
\hat{\theta}=\frac{n}{\sum_{i=1}^{n} \frac{\left(\frac{x_{i}}{\gamma}\right)^{c}}{\alpha+\beta\left(\frac{x_{i}}{\gamma}\right)^{c}}}-\beta .
$$

When $\beta, \theta, \gamma$, and $\mathrm{c}$ are known, it follows from equation (31) that

$$
\hat{\alpha}=-\frac{\theta}{\beta+\theta}+\frac{\theta}{n} \sum_{i=1}^{n}\left(\frac{x_{i}}{\gamma}\right)^{c}-1 \text {. }
$$

When $\alpha, \theta, \gamma$, and $\mathrm{c}$ are known, it follows from equation (31) that

$$
\hat{\beta}=\frac{-n}{\frac{n(\alpha+1)}{\theta}-\sum_{i=1}^{n}\left(\frac{x_{i}}{\gamma}\right)^{c}}-\theta \text {. }
$$

\subsection{Fisher Information}

Let $\boldsymbol{\theta}=\left(\theta_{1}, \theta_{2}, \theta_{3}, \theta_{4}, \theta_{5}\right)=(\alpha, \beta, \theta, \gamma, c)$, and $\operatorname{g}_{L G L W}(x ; \boldsymbol{\theta})$ the LGLW pdf. If $\log \left(g_{L G L W}(x ; \boldsymbol{\theta})\right)$ is twice differentiable with respect to $\boldsymbol{\theta}$, and under certain regularity conditions, Fisher information matrix (FIM) is the $5 \times 5$ matrix whose elements are:

$$
\mathrm{I}(\boldsymbol{\theta})=-\mathrm{E}_{\theta}\left[\frac{\partial^{2} \log \left(g_{L G L W}(X ; \boldsymbol{\theta})\right)}{\partial \theta_{i} \partial \theta_{j}}\right] .
$$

The second and mixed partial derivatives of the log-likelihood function used to obtain the observed Fisher information matrix can be readily computed.

\subsection{Asymptotic Confidence Intervals}

The $5 \times 5$ observed information matrix $\mathbf{J}(\boldsymbol{\theta})=-\frac{\partial^{2} l(\boldsymbol{\theta})}{\partial \boldsymbol{\theta} \partial \boldsymbol{\theta}^{T}}$ can be used for interval estimation of $\alpha, \beta, \theta, \gamma$, and $c$, and for test of hypothesis on these parameters. Under conditions that are 
fulfilled for parameters in the interior of the parameter space but not on the boundary, the asymptotic distribution of $\widehat{\boldsymbol{\theta}}-\boldsymbol{\theta}$ can be approximated by $\mathrm{N}_{5}\left(0, \boldsymbol{J}(\widehat{\boldsymbol{\theta}})^{-1}\right)$. Thus, the multivariate normal $\mathrm{N}_{5}\left(0, \boldsymbol{J}(\widehat{\boldsymbol{\theta}})^{-1}\right)$ distribution can be used to construct approximate confidence intervals and confidence regions for the parameters. In fact, the asymptotic $100(1-\eta) \%$ confidence intervals for $\alpha, \beta, \theta, \gamma$ and $\mathrm{c}$ are given by $\hat{\alpha} \pm Z_{\frac{\eta}{2}} \times \sqrt{I_{\alpha \alpha}^{-1}(\widehat{\boldsymbol{\theta}})}, \hat{\beta} \pm Z_{\frac{\eta}{2}} \times \sqrt{I_{\beta \beta}^{-1}(\widehat{\boldsymbol{\theta}})}, \hat{\theta} \pm Z_{\frac{\eta}{2}} \times$ $\sqrt{I_{\theta \theta}^{-1}(\widehat{\boldsymbol{\theta}})}, \hat{\gamma} \pm Z_{\frac{\eta}{2}} \times \sqrt{I_{\gamma \gamma}^{-1}(\widehat{\boldsymbol{\theta}})}$, and $\hat{c} \pm Z_{\frac{\eta}{2}} \times \sqrt{I_{c c}^{-1}(\widehat{\boldsymbol{\theta}})}$, where $Z_{\frac{\eta}{2}}$ is the $\left(1-\frac{\eta}{2}\right)^{t h}$ quantile of the standard normal distribution.

The likelihood ratio (LR) statistic is useful for testing the goodness-of-fit of the LGLW model and for comparing it with other sub-models such as generalized Lindley (GL) and Lindley (L) distributions. We can easily check if the fit using LGLW model is statistically "superior" to a fit using the GL model for a given data set by computing $\mathrm{w}=2\{l(\hat{\alpha}, \hat{\beta}, \hat{\theta}, \hat{\gamma}, \hat{c})-l(\tilde{\alpha}, \tilde{\beta}, \tilde{\theta}, 1,1)\}$, where $\hat{\alpha}, \hat{\beta}, \hat{\theta}, \hat{\gamma}$, and $\hat{c}$ are the unrestricted MLEs and $\tilde{\alpha}, \tilde{\beta}$, and $\tilde{\theta}$ are the restricted estimates. Also, the LR statistic is asymptotically distributed under the null model as $\chi_{2}^{2}$. Further, the LR test rejects the null hypothesis if $\omega>\xi_{\eta}$, where $\xi_{\eta}$ denotes the upper $100 \eta \%$ point of the $\chi_{2}^{2}$ distribution.

\section{Applications}

The maximum likelihood estimates (MLEs) of the parameters are obtained via the subroutine NLP in SAS. The maximum likelihood estimates (MLEs) of the parameters are obtained via the subroutine NLP in SAS. The estimates (standard error in parenthesis), -2 Log Likelihood, Akaike Information Criteria (AIC), Consistent Akaike Information Criterion (AICC), Bayesian Information Criterion (BIC), SS and KS values are given, where AIC $=2 p-2 \ln L$, AICC $=$ $\mathrm{AIC}+2 \frac{\mathrm{p}(\mathrm{p}+1)}{n-p-1}, \mathrm{BIC}=\mathrm{p} \ln n-2 \ln L$, and $\mathrm{KS}=\max _{1 \leq \mathrm{j} \leq \mathrm{n}}\left\{\mathrm{G}_{\mathrm{LGLW}}\left(x_{(j)}\right)-\frac{j-1}{n}, \frac{j}{n}-G_{L G L W}\left(x_{(j)}\right)\right\}$, where $\mathrm{L}(\hat{\theta})=\mathrm{L}$ is the value of the likelihood function evaluated at the estimated parameters, $\mathrm{n}$ is the number of observations, and $\mathrm{p}$ is the number of estimated parameters are given in Tables 1,2 and 3.

Probability plots (Chambers et al., 1983) are also presented in Figure 4, Figure 5 and Figure 6. For the probability plot, we plotted the estimated cdf $\mathrm{G}_{\mathrm{LGLW}}\left(x_{(j)} ; \hat{\alpha}, \hat{\beta}, \hat{\theta}, \hat{\gamma}, \hat{c}\right)$ against $\frac{j-0.375}{n+0.25}, \mathrm{j}=1,2, \ldots, \mathrm{n}$, where $\mathrm{x}_{(j)}$ are the ordered values of the observed data. We also computed a measure of closeness of each plot to the diagonal line. This measure of closeness is given by the sum of squares $\mathrm{SS}=\sum_{j=1}^{n}\left[\mathrm{G}_{\mathrm{LGLW}}\left(x_{(j)} ; \hat{\alpha}, \hat{\beta}, \hat{\theta}, \hat{\gamma}, \hat{c}\right)-\left(\frac{j-0.375}{n+0.25}\right)\right]^{2}$.

This first data (Aarset, 1987) consists of the times to failure of 50 devices put on life test at

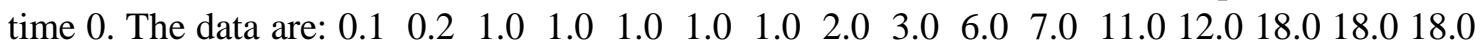


18.018 .021 .032 .036 .040 .045 .046 .047 .050 .055 .060 .063 .063 .067 .067 .067 .067 .072 .0 75.0 79.0 82.0 82.0 83.0 84.0 84.0 84.0 85.0 85.0 85.0 85.0 85.0 86.0 86.0. The results are given in Table 1, and plots are given in Figure 4. The estimated covariance matrix for the LGLW distribution is given by:

$$
\left(\begin{array}{ccccc}
0.000170055 & -1.71 E-10 & -3.18 E-10 & -1.50 E-09 & 2.72 E-06 \\
-1.71 E-10 & 2.19 E-15 & 2.67 E-15 & 1.51 E-15 & -2.73 E-12 \\
-3.18 E-10 & 2.67 E-15 & 3.35 E-15 & 2.81 E-15 & -5.08 E-12 \\
-1.50 E-09 & 1.51 E-15 & 2.81 E-15 & 1.32 E-14 & -2.39 E-11 \\
2.72 E-06 & -2.73 E-12 & -5.08 E-12 & -2.39 E-11 & 4.34 E-08
\end{array}\right)
$$

The 95\% asymptotic confidence intervals are: $\alpha \in 0.087773 \pm 1.96(0.013041), \theta \in$ $0.000000176 \pm 1.96(0.00000005), \beta \in 0.000000161 \pm 1.96(0.00000006), \gamma \in$ $3.597705 \pm 1.96(0.0000001), c \in 5.089304 \pm 1.96(0.000208)$.

The LR test statistics of the hypotheses $H_{0}: \operatorname{LGLW}(\alpha, \theta, 1,1, c)$ vs $\mathrm{H}_{\mathrm{a}}: \operatorname{LGLW}(\alpha, \theta, \beta, \gamma, c)$, $\mathrm{H}_{0}: \operatorname{LGLW}(\alpha, \theta, \beta, \gamma, 1) \quad$ vs $\quad \mathrm{H}_{\mathrm{a}}: \operatorname{LGLW}(\alpha, \theta, \beta, \gamma, \mathrm{c}) \quad$ and $\mathrm{H}_{0}: \operatorname{LGLW}(1, \theta, \beta, \gamma, \mathrm{c}) \quad$ vs $\mathrm{H}_{\mathrm{a}}$ : $\operatorname{LGLW}(\alpha, \theta, \beta, \gamma, \mathrm{c})$ are 43.2 (p-value <0.0001), 34.9 (p-value <0.0001) and 40.5 (p-value $<0.0001)$. We conclude $\operatorname{LGLW}(\alpha, \theta, \beta, \gamma, c)$ distribution is significantly better than the submodels. Also, $\operatorname{LGLW}(\alpha, \theta, \beta, \gamma, c)$ distribution gives the smallest AIC, AICC, BIC, SS and KS values. Consequently, we conclude that the $\operatorname{LGLW}(\alpha, \theta, \beta, \gamma, c)$ distribution is the "best" model for Aarset data.

The second data set given by Murthy et al. (2004) consists of the failure times of 20 mechanical components. The data are: 0.0670 .0680 .0760 .0810 .0840 .0850 .0850 .0860 .089 0.0980 .0980 .1140 .1140 .1150 .1210 .1250 .1310 .1490 .1600 .485 . The results and plots are given in Table 2 and Figure 5. The estimated covariance matrix for the LGLW distribution is given by:

$$
\left(\begin{array}{lllll}
110.73486 & 30.80945 & -13.41218 & 0.02441 & 0.81060 \\
30.80945 & 8.64857 & -3.68959 & 0.00684 & 0.22654 \\
-13.41218 & -3.68959 & 1.66015 & -0.00294 & -0.09848 \\
0.02441 & 0.00684 & -0.00294 & 0.00001 & 0.00018 \\
0.81060 & 0.22654 & -0.09848 & 0.00018 & 0.00600
\end{array}\right)
$$

The LR test statistics of the hypotheses $\mathrm{H}_{0}: \operatorname{LGLW}(\alpha, \theta, \beta, \gamma, 1)$ vs $\mathrm{H}_{\mathrm{a}}$ : $\operatorname{LGLW}(\alpha, \theta, \beta, \gamma, \mathrm{c})$ and $\mathrm{H}_{0}: \operatorname{LGLW}(1, \theta, \beta, \gamma, \mathrm{c})$ vs $\mathrm{H}_{\mathrm{a}}: \operatorname{LGLW}(\alpha, \theta, \beta, \gamma, \mathrm{c})$ are 4.9 (p-value $\left.=0.027\right)$ and 11.8 (p-value $<0.001)$. Therefore, we conclude that the $\operatorname{LGLW}(\alpha, \theta, \beta, \gamma, \mathrm{c})$ distribution is significantly better than the $\operatorname{LGLW}(\alpha, \theta, \beta, \gamma, 1)$ and $\operatorname{LGLW}(1, \theta, \beta, \gamma, c)$ sub-models. Also, note that the $\operatorname{LGLW}(\alpha, \theta, \beta, \gamma, c)$ distribution gives the smallest SS, KS values and second smallest AIC, AICC, BIC values when compare to gamma distribution. We conclude that the $\operatorname{LGLW}(\alpha, \theta, \beta, \gamma, c)$ distribution is a reasonably good model for the failure times data. 
The third example consists of prices $\left(\times 10^{4}\right.$ dollars $)$ of 428 new vehicles for the 2004 year (Kiplinger's Personal Finance, Dec 2003). The data are given in Table 3.

The results and plots are given in Table 4 and Figure 6 . The estimated covariance matrix for the LGLW distribution is given by:

$$
\left(\begin{array}{ccccc}
0.035560103 & -4.29 \mathrm{E}-06 & -4.69 \mathrm{E}-06 & -0.000031261 & 0.001043554 \\
-4.29 \mathrm{E}-06 & 7.66 \mathrm{E}-10 & 5.69 \mathrm{E}-10 & 5.50 \mathrm{E}-09 & -7.53 \mathrm{E}-08 \\
-4.69 \mathrm{E}-06 & 5.69 \mathrm{E}-10 & 6.20 \mathrm{E}-10 & 4.15 \mathrm{E}-09 & -1.36 \mathrm{E}-07 \\
-0.000031261 & 5.50 \mathrm{E}-09 & 4.15 \mathrm{E}-09 & 3.98 \mathrm{E}-08 & -4.91 \mathrm{E}-07 \\
0.001043554 & -7.53 \mathrm{E}-08 & -1.36 \mathrm{E}-07 & -4.91 \mathrm{E}-07 & 0.000072061
\end{array}\right)
$$

Plots of the fitted densities and the histogram, observed probability vs predicted probability, and empirical survival function are given in Figure 6.

The LR test statistics of the hypotheses $\mathrm{H}_{0}: \operatorname{LGLW}(\alpha, \theta, \beta, \gamma, 1)$ vs $\mathrm{H}_{\mathrm{a}}: \operatorname{LGLW}(\alpha, \theta, \beta, \gamma, \mathrm{c})$ and $\mathrm{H}_{0}$ : $\operatorname{LGLW}(1, \theta, \beta, \gamma, \mathrm{c})$ vs $\mathrm{H}_{\mathrm{a}}$ : $\operatorname{LGLW}(\alpha, \theta, \beta, \gamma, \mathrm{c})$ are 46.0 (p-value <0.0001) and 12.6 (pvalue $=0.0004)$, respectively. We conclude that the $\operatorname{LGLW}(\alpha, \theta, \beta, \gamma, c)$ distribution is significantly better than the sub-models. Also, gamma distribution gives the smallest AIC, AICC, BIC, SS, KS values followed by the $\operatorname{LGLW}(\alpha, \theta, \beta, \gamma, \mathrm{c})$ distribution. Consequently, the gamma and $\operatorname{LGLW}(\alpha, \theta, \beta, \gamma, c)$ distributions are good models for prices of 2004 new cars and trucks data.

\section{Concluding Remarks}

In line with results on generalized distributions and following the contents of the T-X class of distributions (Alzaatreh et al., 2013), we derive and present the mathematical and statistical properties of a new generalized Lindley distribution called log generalized Lindley-Weibull (LGLW) distribution. This distribution contains several sub-models including Lindley distribution and the generalized Lindley distribution of Zakerzadeh and Dolati (2009). The hazard rate function of the LGLW distribution can be decreasing, decreasing or bathtub shaped. Moments and distributions of functions of random variables from the LGLW distribution are derived. Uncertainty measures including generalized entropy, Rényi and Shannon entropies are obtained. We discuss maximum likelihood estimation and hypotheses tests of the model parameters. The LGLW distribution permits testing the goodness-of-fit of Lindley and generalized Lindley distribution by taking these distributions as sub-models. Asymptotic confidence intervals for the parameters of the LGLW distribution are given. We fit the LGLW distribution and its sub-models to three real data sets to demonstrate the potential importance, practical relevance and applicability of this model in lifetime analysis and other areas.

\section{Acknowledgments}

The authors thank the associate editor and anonymous referees for careful review of the manuscript and their suggestions and comments which lead to the improvement of the quality of the paper. 
Table 1: LGLW Estimates of Models for Aarset Data

\begin{tabular}{|c|c|c|c|c|c|c|c|c|c|c|c|}
\hline & \multicolumn{5}{|c|}{ Estimates } & \multicolumn{6}{|c|}{ Statistics } \\
\hline & $\alpha$ & $\Theta$ & $\beta$ & $\gamma$ & $c$ & -2 Log Likelihood & AIC & AICC & $\mathrm{BIC}$ & SS & KS \\
\hline \multirow[t]{2}{*}{$\operatorname{GLW}(\alpha, \theta, \beta, \gamma, \mathrm{c})$} & 0.0878 & 0.0000002 & 0.0000002 & 3.5977 & 5.0893 & 439.0 & 449.0 & 450.4 & 458.6 & 0.1055 & 0.1259 \\
\hline & $(0.013041)$ & $(0.00000005)$ & $(0.00000006)$ & $(0.0000001)$ & $(0.0002)$ & & & & & & \\
\hline \multirow[t]{2}{*}{$\operatorname{GLW}(\alpha, \theta, 1,1, c)$} & 0.3081 & 0.0475 & 1 & 1 & 0.8688 & 482.2 & 488.2 & 488.7 & 493.9 & 0.5024 & 0.1893 \\
\hline & $(0.153033)$ & $(0.043501)$ & - & - & $(0.181765)$ & & & & & & \\
\hline \multirow[t]{2}{*}{$\operatorname{GLW}(\alpha, \theta, \beta, \gamma, 1)$} & 0.5281 & 0.0119 & 0.0241 & 0.4525 & 1 & 473.9 & 481.9 & 482.8 & 489.6 & 0.4273 & 0.1790 \\
\hline & $(0.094401)$ & $(0.001106)$ & $(0.010042)$ & $(0.000549)$ & - & & & & & & \\
\hline \multirow[t]{3}{*}{$\operatorname{GLW}(1, \theta, \beta, \gamma, c)$} & 1 & 0.0258 & 0.0279 & 0.4307 & 0.8817 & 479.5 & 487.5 & 488.4 & 495.2 & 0.4813 & 0.1849 \\
\hline & - & $(0.020363)$ & $(0.017642)$ & $(0.002066)$ & (0.150219) & & & & & & \\
\hline & $\lambda$ & $k$ & & & & & & & & & \\
\hline \multirow[t]{3}{*}{$\operatorname{Weibull}(\lambda, \mathrm{k})$} & 44.9125 & 0.9490 & & & & 482.0 & 486.0 & 486.3 & 489.8 & 0.5289 & 0.1928 \\
\hline & (6.902622) & $(0.167396)$ & & & & & & & & & \\
\hline & $\alpha$ & B & & & & & & & & & \\
\hline \multirow[t]{2}{*}{$\operatorname{Gamma}(\alpha, \beta)$} & 0.7991 & 0.0175 & & & & 480.4 & 484.4 & 484.6 & 488.2 & 0.5545 & 0.2022 \\
\hline & (0.163869) & $(0.002753)$ & & & & & & & & & \\
\hline
\end{tabular}

Table 2: LGLW Estimates of Models for Failure Times Data

\begin{tabular}{|c|c|c|c|c|c|c|c|c|c|c|c|}
\hline & \multicolumn{5}{|c|}{ Estimates } & \multicolumn{6}{|c|}{ Statistics } \\
\hline & $\alpha$ & $\Theta$ & $\beta$ & $\gamma$ & c & -2 Log Likelihood & AIC & AICC & $\mathrm{BIC}$ & SS & KS \\
\hline \multirow[t]{2}{*}{$\operatorname{GLW}(\alpha, \theta, \beta, \gamma, \mathrm{c})$} & 37.885305 & 12.73676 & 3.915639 & 0.005146 & 0.352967 & -64.7 & -54.7 & -50.4 & -49.7 & 0.2453 & 0.2165 \\
\hline & $(10.5231)$ & $(2.9408)$ & $(1.2885)$ & $(0.0023)$ & $(0.0775)$ & & & & & & \\
\hline \multirow[t]{2}{*}{$\operatorname{GLW}(\alpha, \theta, \beta, \gamma, 1)$} & 3.3948 & 0.000001956 & 4.8031 & $5.41 \mathrm{E}-08$ & 1 & -59.8 & -51.8 & -49.1 & -47.8 & 0.2707 & 0.2251 \\
\hline & $(1.62 \mathrm{E}-18)$ & (3.64E-12) & $(1.28 \mathrm{E}-25)$ & $(1.32 \mathrm{E}-10)$ & - & & & & & & \\
\hline \multirow[t]{3}{*}{$\operatorname{GLW}(1, \theta, \beta, \gamma, c)$} & 1 & 5.3422 & $1.66 \mathrm{E}-07$ & 0.3817 & 1.6422 & -52.8 & -44.8 & -42.2 & -40.9 & 0.4142 & 0.2641 \\
\hline & - & $(0.0063)$ & (1.109E-05) & $(0.1446)$ & $(0.3491)$ & & & & & & \\
\hline & $\lambda$ & k & & & & & & & & & \\
\hline \multirow[t]{3}{*}{$\operatorname{Weibull}(\lambda, \mathrm{k})$} & 0.1376 & 1.6422 & & & & -52.8 & -48.8 & -48.1 & -46.9 & 0.4142 & 0.2641 \\
\hline & $(0.0226)$ & $(0.329)$ & & & & & & & & & \\
\hline & $\alpha$ & $\mathrm{B}$ & & & & & & & & & \\
\hline \multirow[t]{2}{*}{$\operatorname{Gamma}(\alpha, \beta)$} & 4.2441 & 34.9163 & & & & -59.8 & -55.8 & -55.1 & -53.8 & 0.2722 & 0.2253 \\
\hline & $(2.7031)$ & $(27.7806)$ & & & & & & & & & \\
\hline
\end{tabular}


Broderick O. Oluyede, Fedelis Mutiso, Shujiao Huang

299

Table 3: Prices of 2004 New Cars and Trucks Data

\begin{tabular}{|c|c|c|c|c|c|c|c|c|c|c|c|c|c|c|}
\hline 1.028 & 1.0539 & 1.076 & 1.0995 & 1.1155 & 1.129 & 1.156 & 1.169 & 1.1839 & 1.1905 & 1.1939 & 1.2269 & 1.236 & 1.2585 & 1.274 \\
\hline 1.28 & 1.2884 & 1.2965 & 1.327 & 1.327 & 1.358 & 1.367 & 1.373 & 1.3839 & 1.4085 & 1.4165 & 1.417 & 1.43 & 1.4385 & 1.45 \\
\hline 1.461 & 1.4622 & 1.463 & 1.474 & 1.481 & 1.484 & 1.485 & 1.503 & 1.504 & 1.5295 & 1.5389 & 1.5389 & 1.546 & 1.5495 & 1.55 \\
\hline 1.5568 & 1.558 & 1.5825 & 1.585 & 1.604 & 1.635 & 1.6385 & 1.6495 & 1.6497 & 1.653 & 1.6695 & 1.6722 & 1.6999 & 1.7045 & 1.7163 \\
\hline 1.72 & 1.7232 & 1.7262 & 1.7475 & 1.7495 & 1.763 & 1.764 & 1.7735 & 1.775 & 1.7985 & 1.8345 & 1.8435 & 1.869 & 1.8715 & 1.8739 \\
\hline 1.876 & 1.882 & 1.8825 & 1.8892 & 1.8995 & 1.9005 & 1.909 & 1.911 & 1.9135 & 1.924 & 1.927 & 1.9312 & 1.9339 & 1.9479 & 1.949 \\
\hline 1.956 & 1.9635 & 1.9635 & 1.9825 & 1.986 & 1.986 & 1.9945 & 1.9999 & 2.013 & 2.014 & 2.0215 & 2.022 & 2.0255 & 2.029 & 2.03 \\
\hline 2.031 & 2.032 & 2.0339 & 2.037 & 2.0445 & 2.0449 & 2.051 & 2.0585 & 2.0615 & 2.0939 & 2.1055 & 2.1055 & 2.1087 & 2.141 & 2.1445 \\
\hline 2.1589 & 2.1595 & 2.1595 & 2.1795 & 2.1825 & 2.184 & 2.19 & 2.1965 & 2.2 & 2.201 & 2.2035 & 2.218 & 2.2225 & 2.226 & 2.229 \\
\hline 2.235 & 2.2388 & 2.2395 & 2.245 & 2.2515 & 2.257 & 2.2595 & 2.2735 & 2.2775 & 2.3215 & 2.329 & 2.3495 & 2.3495 & 2.356 & 2.3675 \\
\hline 2.3699 & 2.376 & 2.3785 & 2.382 & 2.3845 & 2.3895 & 2.3895 & 2.3955 & 2.413 & 2.4225 & 2.4295 & 2.4345 & 2.452 & 2.4589 & 2.4695 \\
\hline 2.478 & 2.4885 & 2.4895 & 2.495 & 2.4955 & 2.5 & 2.5045 & 2.5092 & 2.513 & 2.5135 & 2.5193 & 2.5215 & 2.5395 & 2.552 & 2.564 \\
\hline 2.5645 & 2.57 & 2.57 & 2.5717 & 2.592 & 2.5935 & 2.594 & 2.5955 & 2.5995 & 2.6 & 2.606 & 2.6135 & 2.6189 & 2.6395 & 2.647 \\
\hline 2.651 & 2.6545 & 2.656 & 2.665 & 2.686 & 2.691 & 2.693 & 2.696 & 2.699 & 2.6992 & 2.702 & 2.7145 & 2.72 & 2.7339 & 2.737 \\
\hline 2.745 & 2.749 & 2.749 & 2.756 & 2.771 & 2.7905 & 2.793 & 2.7995 & 2.8345 & 2.837 & 2.8495 & 2.8495 & 2.8739 & 2.875 & 2.879 \\
\hline 2.88 & 2.9282 & 2.9322 & 2.9345 & 2.938 & 2.944 & 2.9562 & 2.9595 & 2.967 & 2.9795 & 2.9865 & 2.9995 & 2.9995 & 3.0245 & 3.0295 \\
\hline 3.0315 & 3.0492 & 3.0795 & 3.0835 & 3.086 & 3.0895 & 3.092 & 3.095 & 3.1045 & 3.1145 & 3.123 & 3.137 & 3.1545 & 3.1545 & 3.1745 \\
\hline 3.184 & 3.1849 & 3.189 & 3.2235 & 3.2245 & 3.228 & 3.235 & 3.2415 & 3.2445 & 3.2455 & 3.2495 & 3.266 & 3.278 & 3.2845 & 3.3112 \\
\hline 3.318 & 3.3195 & 3.326 & 3.3295 & 3.336 & 3.343 & 3.348 & 3.35 & 3.354 & 3.378 & 3.384 & 3.3895 & 3.3995 & 3.3995 & 3.439 \\
\hline 3.448 & 3.4495 & 3.4495 & 3.456 & 3.4845 & 3.4895 & 3.5105 & 3.5145 & 3.5495 & 3.5515 & 3.5545 & 3.5695 & 3.5725 & 3.592 & 3.594 \\
\hline 3.594 & 3.5995 & 3.61 & 3.6395 & 3.664 & 3.6895 & 3.6945 & 3.6995 & 3.7 & 3.7245 & 3.739 & 3.753 & 3.756 & 3.763 & 3.773 \\
\hline 3.7885 & 3.7895 & 3.7995 & 3.838 & 3.883 & 3.9195 & 3.9235 & 3.925 & 3.9465 & 3.964 & 3.9995 & 4.0095 & 4.0235 & 4.032 & 4.034 \\
\hline 4.0565 & 4.059 & 4.067 & 4.072 & 4.084 & 4.0845 & 4.101 & 4.1045 & 4.125 & 4.1465 & 4.1475 & 4.1815 & 4.1995 & 4.249 & 4.2565 \\
\hline 4.2735 & 4.284 & 4.2845 & 4.2915 & 4.3175 & 4.3365 & 4.3495 & 4.3755 & 4.3895 & 4.424 & 4.4295 & 4.4535 & 4.4925 & 4.4995 & 4.521 \\
\hline 4.5445 & 4.57 & 4.5707 & 4.61 & 4.6265 & 4.647 & 4.6995 & 4.7955 & 4.804 & 4.817 & 4.8195 & 4.845 & 4.909 & 4.969 & 4.9995 \\
\hline 4.9995 & 5.047 & 5.0595 & 5.067 & 5.1535 & 5.212 & 5.2195 & 5.2365 & 5.2545 & 5.2775 & 5.2795 & 5.28 & 5.2975 & 5.4765 & 5.4995 \\
\hline 5.575 & 5.617 & 5.6595 & 5.6665 & 5.727 & 5.9995 & 6.067 & 6.312 & 6.32 & 6.48 & 6.5 & 6.8995 & 6.919 & 6.9195 & 6.9995 \\
\hline 7.225 & 7.3195 & 7.432 & 7.4995 & 7.4995 & 7.5 & 7.62 & 7.6765 & 7.687 & 7.9165 & 8.1795 & 8.1995 & 8.4165 & 8.46 & 8.697 \\
\hline 8.6995 & 8.9765 & 9.052 & 9.482 & 12.177 & 12.667 & 12.842 & 19.2465 & & & & & & & \\
\hline
\end{tabular}


Table 4: LGLW Estimates of Models for Prices of 2004 New Cars and Trucks Data

\begin{tabular}{|c|c|c|c|c|c|c|c|c|c|c|c|}
\hline & \multicolumn{5}{|c|}{ Estimates } & \multicolumn{6}{|c|}{ Statistics } \\
\hline & $\alpha$ & $\Theta$ & $\beta$ & $\gamma$ & c & $\begin{array}{c}-2 \text { Log } \\
\text { Likelihood }\end{array}$ & AIC & AICC & $\mathrm{BIC}$ & SS & KS \\
\hline \multirow[t]{2}{*}{$\operatorname{GLW}(\alpha, \theta, \beta, \gamma, c)$} & 1.5404 & 0.0001 & 2.0009 & 0.0006 & 1.1998 & 1573.2 & 1583.2 & 1583.4 & 1603.5 & 0.9086 & 0.0760 \\
\hline & $(0.1886)$ & $(0.00003)$ & $(0.00003)$ & $(0.0002)$ & $(0.008)$ & & & & & & \\
\hline \multirow[t]{2}{*}{$\operatorname{GLW}(\alpha, \theta, \beta, \gamma, 1)$} & 1.3017 & $2.53 \mathrm{E}-07$ & 2.00 & $3.61 \mathrm{E}-07$ & 1 & 1619.2 & 1627.2 & 1627.3 & 1643.4 & 2.1103 & 0.1300 \\
\hline & $(4.73 \mathrm{E}-17)$ & $(4.29 E-10)$ & $(2.68 \mathrm{E}-24)$ & $(3.01 \mathrm{E}-10)$ & - & & & & & & \\
\hline \multirow[t]{3}{*}{$\operatorname{GLW}(1, \theta, \beta, \gamma, c)$} & 1 & $2.63 \mathrm{E}-04$ & 2.0416 & 0.0044 & 1.3407 & 1585.9 & 1593.9 & 1594.0 & 1610.1 & 1.0395 & 0.0800 \\
\hline & - & $(0.0003)$ & (0.000018) & (0.005) & $(0.07)$ & & & & & & \\
\hline & $\lambda$ & k & & & & & & & & & \\
\hline \multirow[t]{3}{*}{$\operatorname{Weibull}(\lambda, \mathrm{k})$} & 3.7120 & 1.8390 & & & & 1638.4 & 1642.4 & 1642.4 & 1650.5 & 1.7155 & 0.0989 \\
\hline & (0.11) & $(0.11)$ & & & & & & & & & \\
\hline & A & B & & & & & & & & & \\
\hline \multirow[t]{2}{*}{$\operatorname{Gamma}(\alpha, \beta)$} & 4.0703 & 1.2419 & & & & 1555.4 & 1559.4 & 1559.5 & 1567.6 & 0.6973 & 0.0688 \\
\hline & (0.35) & $(0.13)$ & & & & & & & & & \\
\hline
\end{tabular}




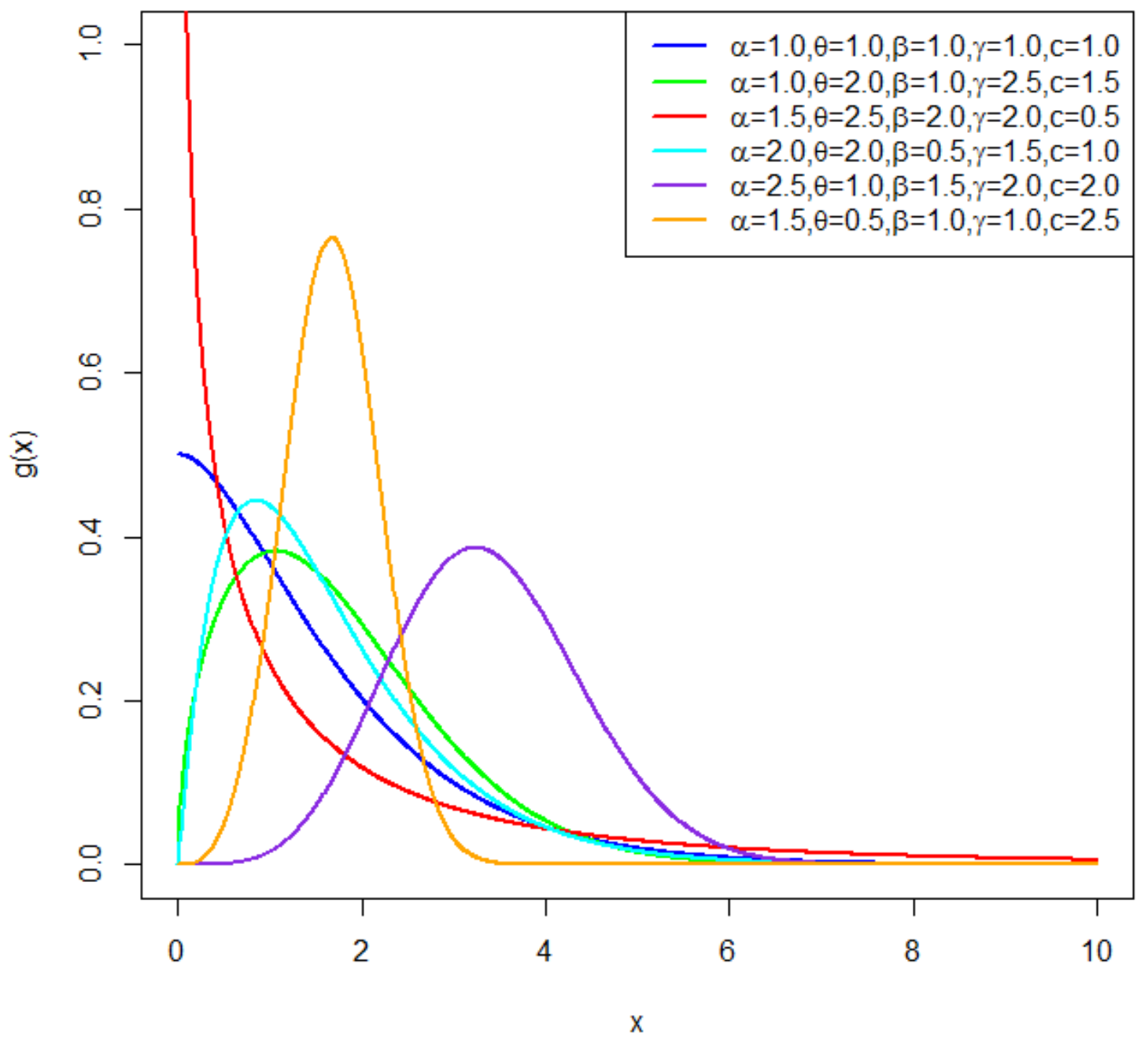

Figure 1: Plot of the pdf of LGLW distribution 


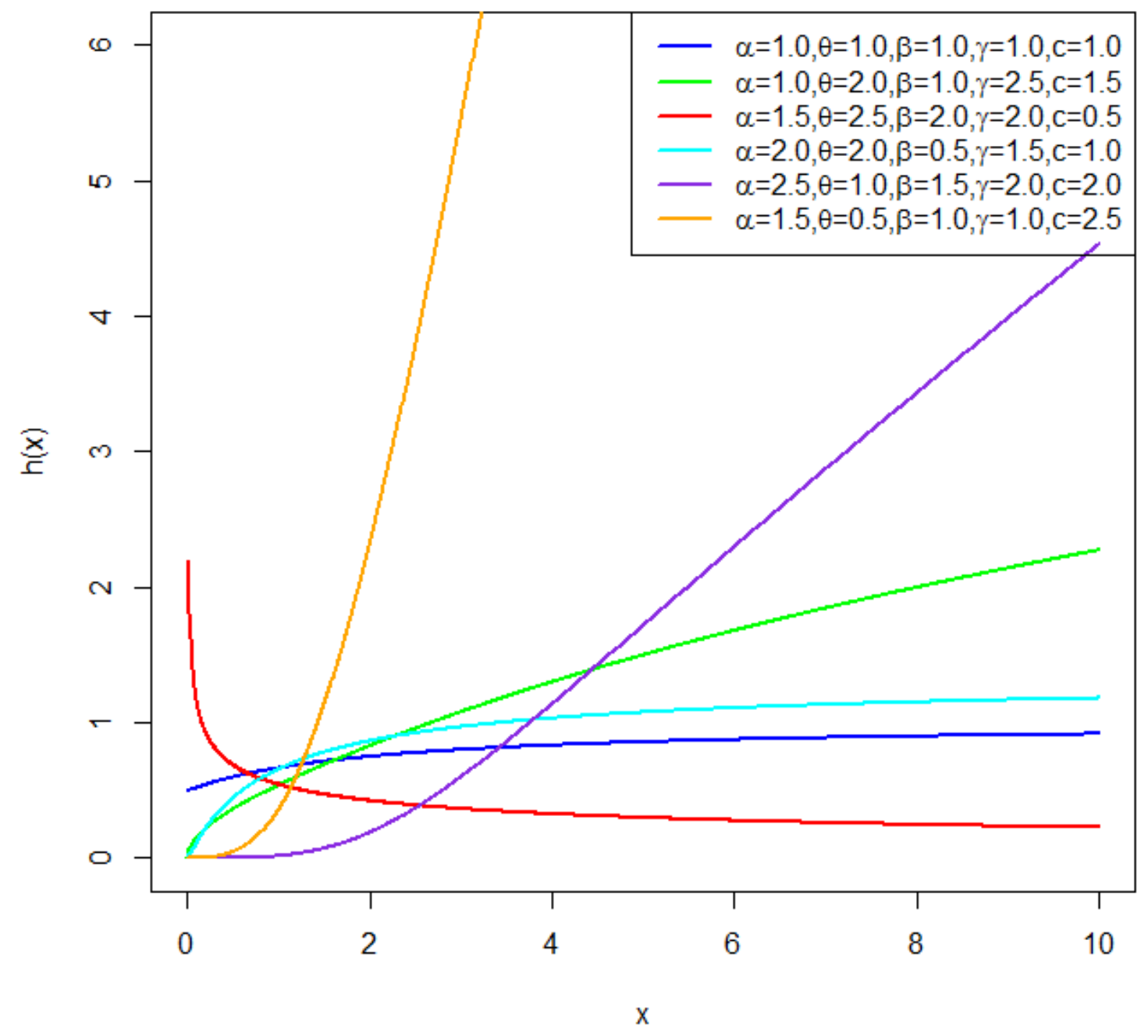

Figure 2: Plot of Hazard Function 


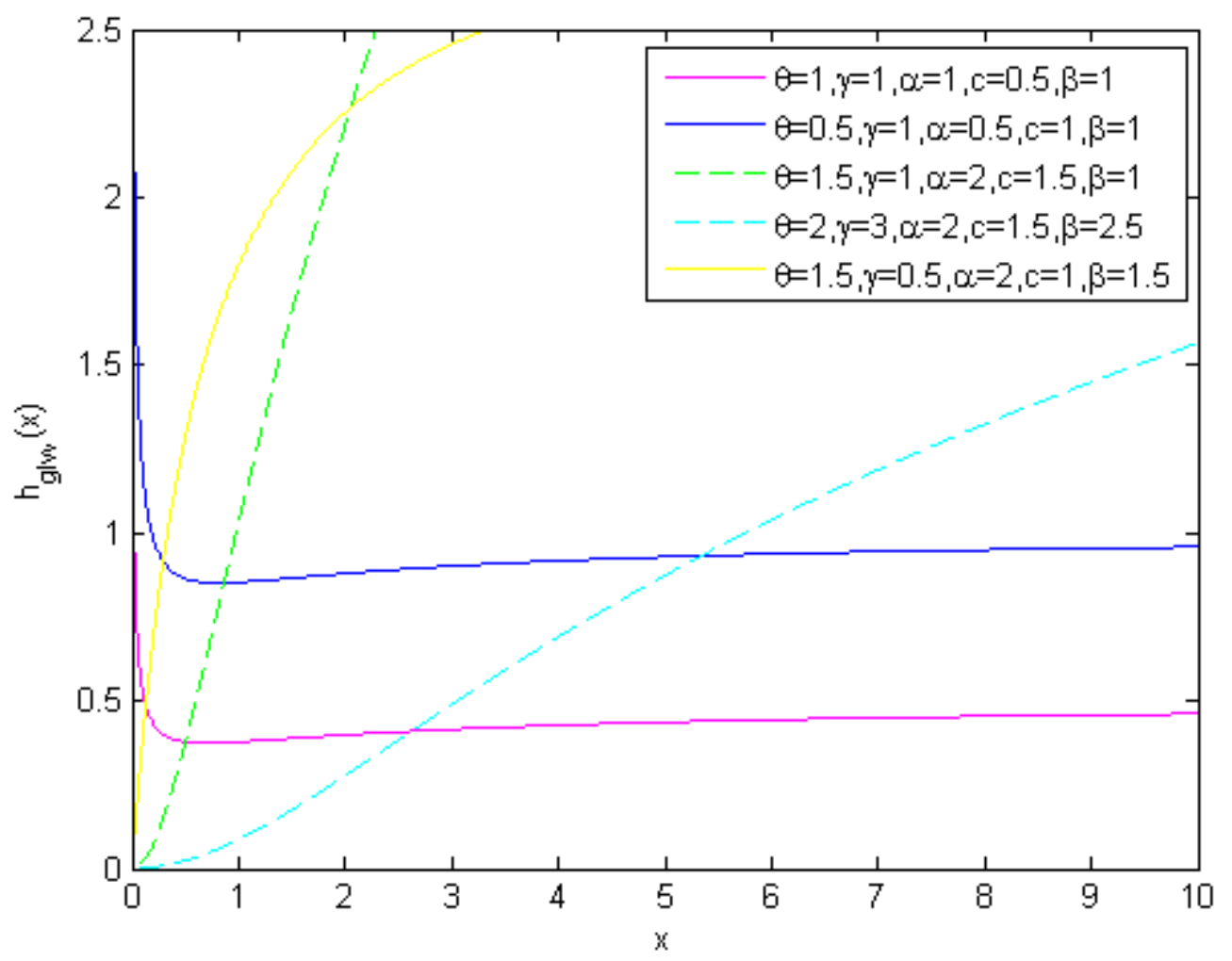

Figure 3: Plot of Hazard Function 

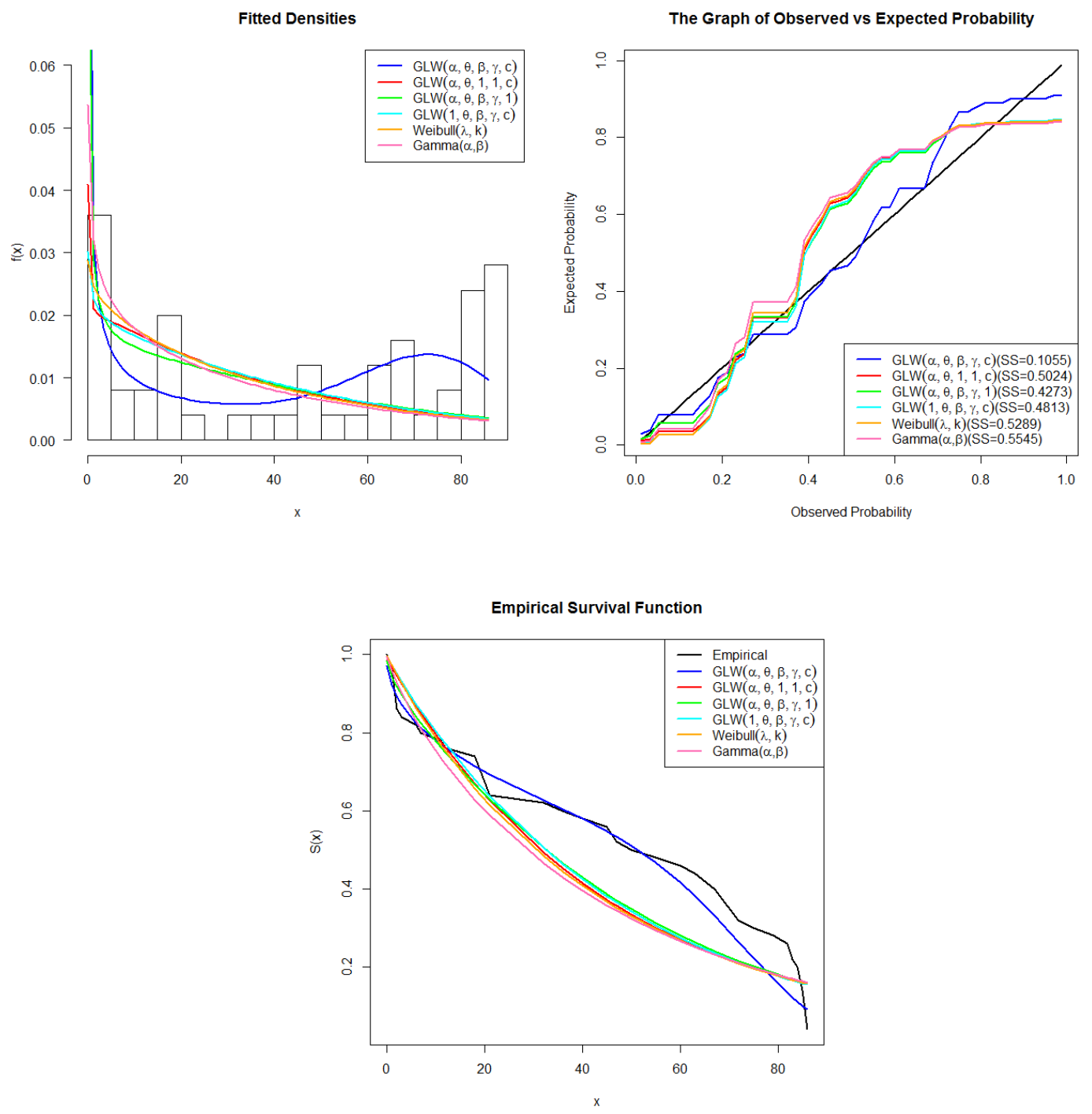

Figure 4: Fitted Densities, Observed Probabilities, and Empirical Survival Functions Plots for Aarset Data 

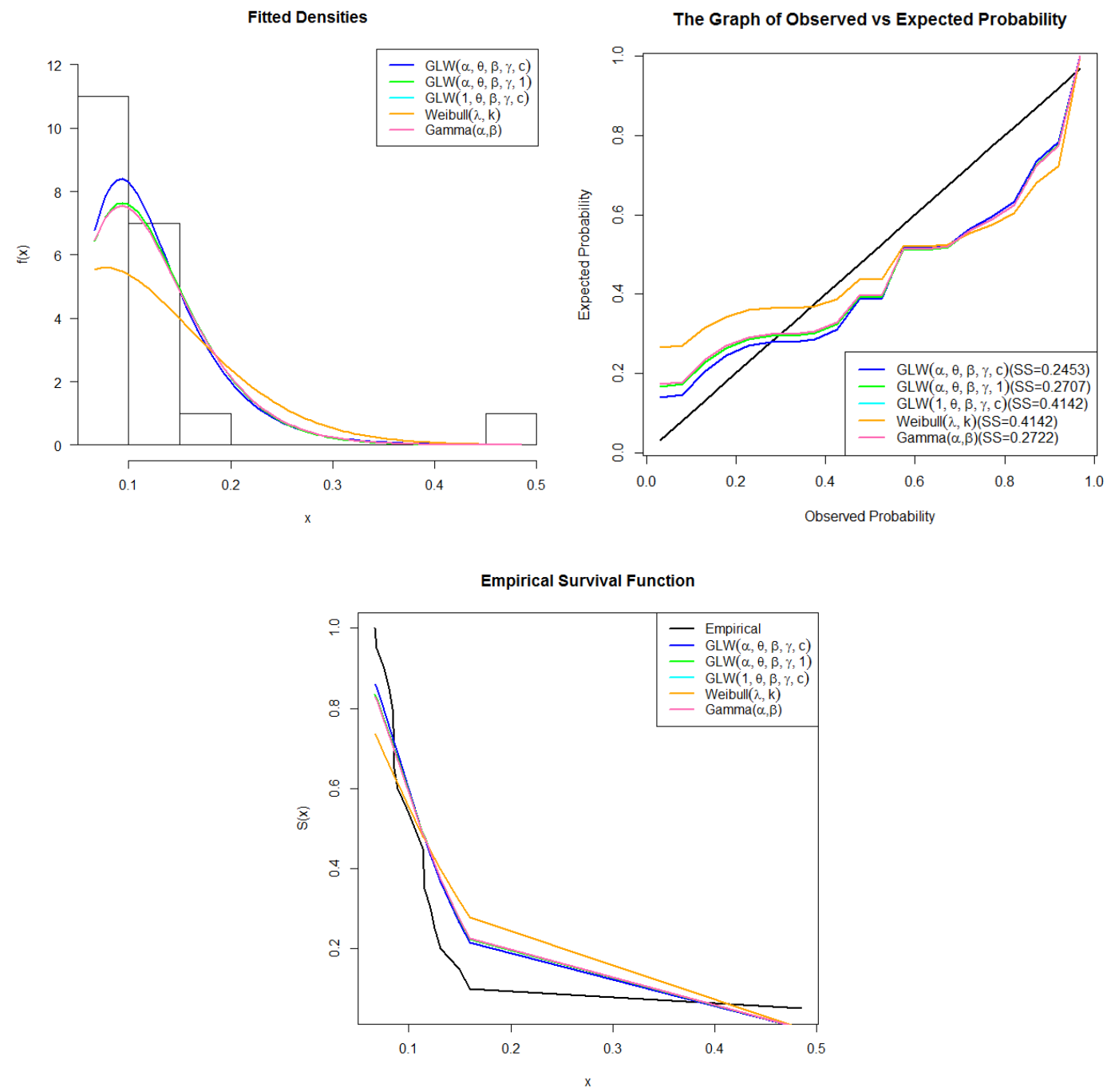

Figure 5: Fitted Densities, Observed Probabilities, and Empirical Survival Functions Plots for Failure Times Data 

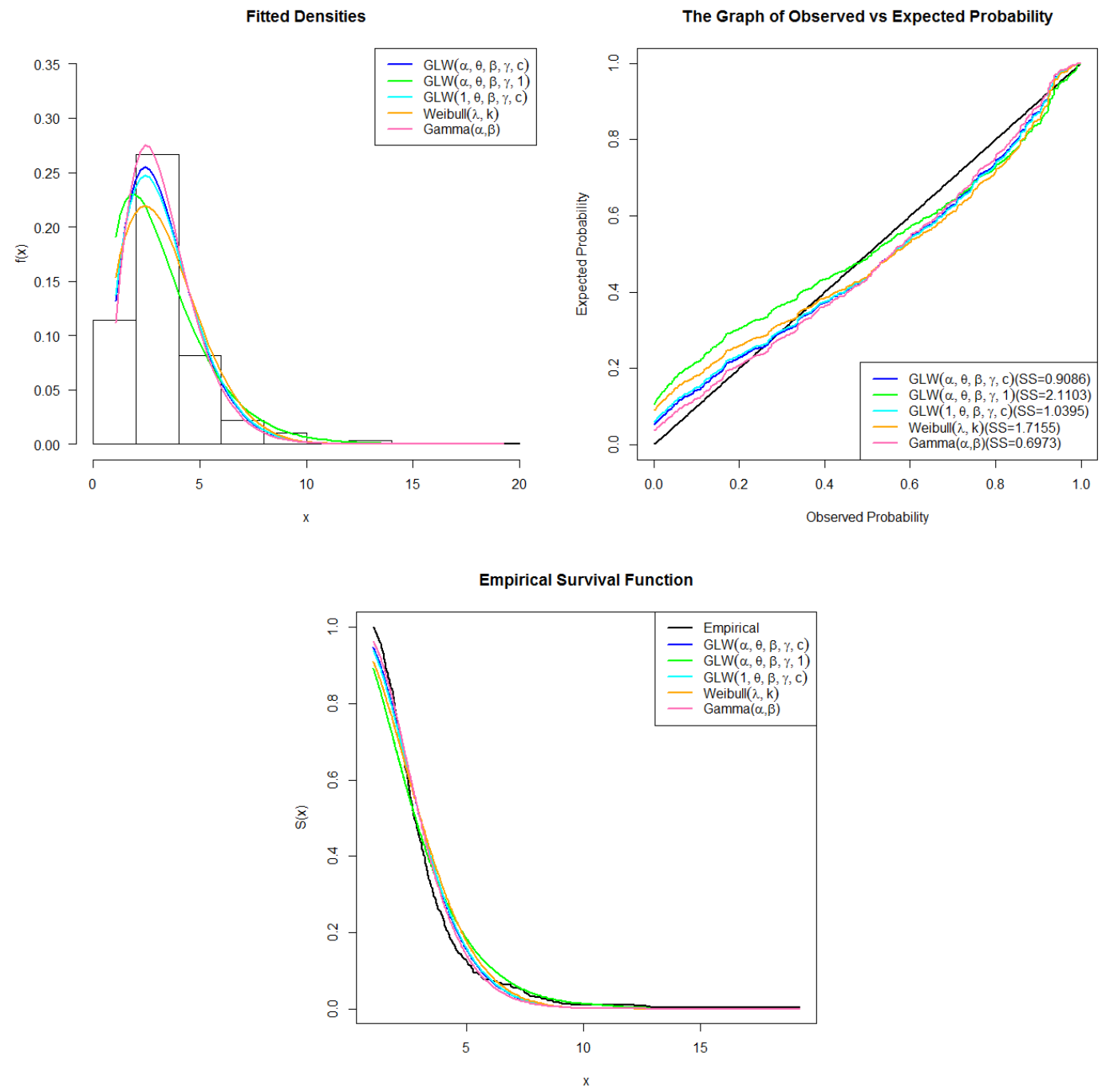

Figure 6: Fitted Densities, Observed Probabilities, and Empirical Survival Functions Plots for Prices of 2004 New Cars and Trucks Data 


\section{References}

[1] Aarset, M. V. (1987). How to Identify Bathtub Hazard Rate, IEEE Transactions on Reliability, 36(1), 106-108.

[1] Aarset, M. V. (1987). How to Identify Bathtub Hazard Rate, IEEE Transactions on Reliability, 36(1), 106-108.

[2] Alzaatreh, A., Lee, C., and Famoye, F. (2013). A New Method for Generating Families of Continuous Distributions, Metron, 71(1), 63-79.

[3] Bakouch, H. S., Al-Zahrani, B. M., Al-Shomrani, A. A., Marchi, V. A. A., and Louzada, F. (2012). An Extended Lindley Distribution, Journal of the Korean Statistical Society, 41, 75-85.

[4] Chambers, J., Cleveland, W., Kleiner, B. and Tukey, J. (1983). Graphical Methods for Data Analysis, Chapman and Hall, London.

[5] Cordeiro, G. M., Hashimoto, E., M., Ortega, E. M. M, and Pascoa, M. A., R. (2012). The McDonald Extended Distribution: Properties and applications, AstA Advances in Statistical Analysis, 96(3), 409-433, 2012.

[6] Eugene, N., Lee, C., and Famoye, F. (2002). Beta-normal distribution and its applications, Communications in Statistics: Theory and Methods, 31, 497-512.

[7] Famoye, F., Lee, C., and Olumolade, O. (2005). The Beta-Weibull Distribution, Journal of Statistical Theory and Applications, 121-138.

[8] Ghitany, M. E., Alqallaf, F., Al-Mutairi, D. K., and Husain, H. A. (2011). A Two-Parameter Weighted Lindley Distribution and its Applications to Survival Data, Mathematics and Computers in Simulation, 81, 1190-1201.

[9] Ghitany, M. E., Al-Mutairi, D. K., Balakrishnan, N., and Al-Enezi, L. J. (2013). Power Lindley Distribution and Associated Inference, Computational Statistics and Data Analysis, 64, 20-33.

[10] Ghitany, M. E., Atieh, B., and Nadarajah, S. (2008). Lindley Distribution and its Application, Mathematics and Computers in Simulation, 78(4), 493-506.

[11] Glaser, R. E. (1980). Bathtub and related failure rate characterizations, Journal of American Statistical Association, 75, 667-672.

[12] Kleiber, C., and Kotz, S. (2003). Statistical Size Distributions in Economics and Actuarial Sciences, Wiley, New York. 
[13] Krishna, H., and Kumar, K. (2011). Reliability Estimation in Lindley Distribution with Progressively Type II Right Censored Sample, Mathematics and Computers in Simulation, 82, 281-294.

[14] Kumaraswamy, P., (1980). Generalized Probability Density Function for Double-Bounded Random Process, Journal of Hydrology, 46, 79-88.

[15] Lindley, D. V. (1958). Fiducial Distributions and Bayes' Theorem, Journal of the Royal Society, Series B, 20, 102-107.

[16] Murthy, D. N. P., Xie, M., and Jiang, R. (2004). Weibull models, Wiley series in probability and statistics, John Wiley and Sons.

[17] Nadarajah, S., Bakouch, H. S., and Tahmasbi, R. (2011). A generalized Lindley Distribution, Technical Report, School of Mathematics, University of Manchester, UK.

[18] Oluyede, B. O., and Yang, T. (2014). A New class of Generalized Lindley Distribution with Applications, To appear in the Journal of Statistical Computation and Simulation.

[19] Pinho, L. G. B., Cordeiro, G. M., and Nobre, J. S. (2012). The Gamma-Exponentiated Weibull Distribution, Journal of Statistical Theory and Applications, 11(4), 379-395.

[20] Renyi, A. (1961). On Measures of Entropy and Information, Berkeley Symposium on Mathematical Statistics and Probability, 1(1), 547 - 561.

[21] Sankaran, M. (1970). The Discrete Poisson-Lindley distribution, Biometrics, 26, 145-149.

[22] Shannon, E. (1948). A Mathematical Theory of Communication, The Bell System Technical Journal, 27 (10), 379 - 423, 623-656.

[23] Ristić, M. M., and Balakrishnan, N. (2011). The Gamma-Exponentiated Exponential Distribution, Journal of Statistical Computation and Simulation, 82(8), 1191-1206.

[24] Yang, T., and Oluyede, B. O. (2014). The Exponentiated Kumaraswamy Lindley Distribution, Submitted.

[25] Zakerzadeh, H., and Dolati, A. (2009). Generalized Lindley Distribution, Journal of Mathematical Extension, 3(2), 13-25.

[26] Zografos, K., and Balakrishnan, N. (2009). On Families of beta- and Generalized GammaGenerated Distribution and Associated Inference, Statistical Methodology, 6, 344-362. 
Broderick O. Oluyede

Department of Mathematical Sciences

Georgia Southern University, GA 30460, USA

boluyede@georgiasouthern.edu

Fedelis Mutiso

Department of Biostatistics

University of Washington, Seattle, WA 98195-7232, USA

fm00344@uw.edu

Shujiao Huang

Department of Mathematics

University of Houston, Houston, TX 77204-3008, USA

zsh05400@math.uh.edu 
